\title{
Functional Modeling View on Product and Process Engineering in Design and Operations
}

Jørgensen, Sten Bay; Lind, Morten; Jensen, Niels

Published in:

Industrial and Engineering Chemistry Research

Link to article, DOI:

10.1021/acs.iecr.8b06338

Publication date:

2019

Document Version

Peer reviewed version

Link back to DTU Orbit

Citation (APA):

Jørgensen, S. B., Lind, M., \& Jensen, N. (2019). Functional Modeling View on Product and Process Engineering in Design and Operations. Industrial and Engineering Chemistry Research, 58(26), 11129-11148.

https://doi.org/10.1021/acs.iecr.8b06338

\section{General rights}

Copyright and moral rights for the publications made accessible in the public portal are retained by the authors and/or other copyright owners and it is a condition of accessing publications that users recognise and abide by the legal requirements associated with these rights.

- Users may download and print one copy of any publication from the public portal for the purpose of private study or research.

- You may not further distribute the material or use it for any profit-making activity or commercial gain

- You may freely distribute the URL identifying the publication in the public portal 


\title{
Functional Modelling View on Product and Process Engineering in Design and Operations
}

\author{
Sten Bay Jørgensen, ${ }^{* \dagger}$ Morten Lind, ${ }^{\ddagger}$ and Niels Jensen $₫$ \\ $\dagger$ Department of Chemical and Biochemical Engineering, Technical University of Denmark, \\ DK-2800 Kgs.Lyngby, Denmark \\ $\ddagger$ Department of Electrical Engineering, Technical University of Denmark, DK-2800 \\ Kgs.Lyngby, Denmark \\ 【Safepark, Kannikestrcede 14, DK-3550 Slangerup, Denmark \\ E-mail: sbj@kt.dtu.dk
}

\begin{abstract}
Recent significant developments within process systems engineering demonstrate a need for more flexible tools to handle the many diverse model based tasks within product and process synthesis, design, innovation and operation. Consequently it is relevant to have a closer look at one of the modelling methodologies which have emerged. This paper addresses functional modelling for the purpose of the object system under investigation. The purpose of functional modelling is to represent the relations between the system goal and the underlying phenomena.

The role of different aspects of functional modelling within the ongoing development of process systems engineering is analyzed. The fundamental principles of functional modelling for the object under investigation are presented and functional modelling hermeneutics are defined as translating the modelling goal into desired system properties, identifying the needed theory to model the system to represent these properties and identifying criteria for evaluation of system purpose and performance. Different
\end{abstract}


types of functional models are developed leading up to a discussion of their relation to quantitative computational physicochemical models. The application of functional modelling concepts is reviewed first for computer aided molecular design then for process design, innovation and finally for process monitoring including alarm handling. Within process modelling it is demonstrated that functional modelling hermeneutics can be viewed as a common foundation for many different functional model types applied in as diverse areas as monitoring, alarm handling, fault detection and consequence reasoning as well as for traditional simulation with computational physicochemical models. The concepts of interpretation or hermeneutics of modelling and design are suggested as being essential for chemical engineering science. The paper concludes that with the desire for more flexible tools within process systems engineering it may be worthwhile to base such tools on the functional modelling paradigm as a common foundation for the different model types.

\section{Introduction}

The increasing industrial and agricultural activities to nurture and supply the increasing world population demands strong emphasis on sustainability to avoid depletion of nonrenewable resources and increasing environmental load from greenhouse gas emission. Significant improvement is required from the process industry at large to achieve more effective selection and usage of raw materials and energy sources while minimizing waste generation without compromising the economic value to the enterprise. Responding effectively to these challenges require deep insight into the characteristics of a sustainable system. For example, how to incorporate the product and the related supply chain within the system boundary under investigation, how to synthesize and design the product and its manufacturing process and how to monitor and optimize its safe operation. Fortunately, the Process Systems Engineering community is actively developing methods for improving product and process synthesis, design, innovation and monitoring facilitated by many initiatives often as 
collaboration between academia and industry or inspired by industry.

Product and process modelling are fundamental activities for systems engineering. Many different types of models are used throughout the lifespan of a process or product. Product models aim at describing the product thermodynamics and properties from knowledge of the molecular product structure. Process models aims at describing the process functionality for synthesis, design and operation. The process models build on conservation principles and fundamental phenomena. Process models include also models developed for HAZOP and for fault and event analysis as well as models aimed at emergency planning. The importance of the application and the use of model-based methods in the analysis and the operation of chemical/biochemical/pharmaceutical processes has been highlighted by many authors. Most often quantitative computational physicochemical are used to represent the interactions between the underlying phenomena and mechanisms. Development of such computational physicochemical models requires a high level of understanding and a detailed analysis of the phenomena in the different systems. ${ }^{1-3}$ Systematic approaches for the development and improvement of physicochemical models models is therefore very important, especially because the re-use of the mathematical models becomes easier and more efficient. ${ }^{4}$ Recent developments point at a need for new modelling tools to support product and process synthesis and design as well as process operation and monitoring. ${ }^{5}$ Consequently it is desirable to identify and discuss some of the more promising modelling methods within process systems engineering.

The purpose of functional modelling is to represent the relations between the system goal and the underlying phenomena. Functional modelling recently has attracted significant interest within other fields of engineering, in particular product engineering where the need to describe the product function and its manufacturing process is important for obtaining an overall system description to facilitate the communication and understanding between engineers of various disciplines and to obtain means to enable computer based reasoning for decision making. ${ }^{6}$ Vermaas $^{7}$ points out that functional models of technical devices are 
ambiguous since there are few common concepts in engineering. Therefore it is timely that the functional modelling view and concept most used in process engineering is presented, to also seed the discussion of this approach.

The paper first develops the functional modelling methodology and presents how this is applied for modelling different aspects of product and process engineering. In the applications section product modelling and design is reviewed as illustrative examples of functional modelling, before accomplishments within process design and innovation are reviewed in the light of functional modelling. Finally accomplishments within functional modelling for process monitoring are reviewed before potential perspectives are outlined and discussed whereafter the conclusions are drawn. Throughout the present paper synthesis and design is described as design except where otherwise noted.

\section{Functional Modelling Methodology}

Actions of humans and other living species are motivated by urge, need or requirement. In engineering design and analysis the need is represented by the purpose of the intended engineering artifact. Thus understanding the relation between the system and its symbolic representation is directly linked to the ability of the symbolic representation to represent the

purpose of the artifact. This relation is fundamental to systems engineering. ${ }^{8-10}$ Thus for development of a systems approach it is essential to be able to obtain a symbolic representation of the product or process properties for the relevant system purpose. Obtaining a symbolic representation involves understanding the link between the product/process phenomena and behavior and its symbolic representation. Understanding this link involves utilizing or developing a theory for the phenomena exploited by the artifact such that these can be represented symbolically.

Understanding or interpreting the link between an artifact and its symbolic representation has a long history in philosophy where it is called hermeneutics. This name originated 
from the name of the greek messenger god Hermes. For Ricoeur, ${ }^{11}$ hermeneutics (in the adjective form hermeneutics means 'concerning interpretation' and as a noun it means 'a method or theory of interpretation ${ }^{12}$ ) is understanding the link between the things and their symbolic representation, i.e. the logic argumentation between the two. This interplay between the physicochemical and the social frameworks of interpretation is illustrated in Figure 1 where it can be seen as an instance of the hermeneutic circle known from theory of text interpretation. ${ }^{11}$ The interpretation of a text is a circular procedure because the meaning of the words (the parts) depends on the meaning of sentences (the whole) and vice versa. Similarly, the relevance or significance of a physicochemical phenomenon (the part) for a modelling problem depends on system functions and goals i.e. the significance of the whole. Conversely, the goals and the functions of the system cannot be defined without reference to the physicochemical properties. This logic argumentation between the artifact and its symbolic representation for the problem under investigation accordingly may be labelled the modelling hermeneutics.

Wimsatt ${ }^{13}$ discusses functional modelling from several points of view and focus upon three cases of functions: a 'perspectival' function: ... where the functions of an entity in a system are to be evaluated relative to a view or perspective of what the system is doing. As a special case thereof a 'consequence' function:.... where a function of an entity is a consequence of that entity's operation which contributes to the 'proper functioning' of some system of which it is part. And also a 'teleological' function: If some entity has a function in this sense,.. it is useful for or contributes to the attainment of some end or purpose of some user or system. Clearly this is also a subclass of the perspectival case. Through an elaborate discussion Wimsatt ${ }^{13}$ shows that functional analysis and explanation are scientifically objective modes of analysis and further that teleology (which is explanation of phenomena in terms of the purpose they serve rather than of the cause by which they arise ${ }^{14}$ ) has a scientific role in system characterization. Thus functional modelling understood as modelling for the purpose of the object under investigation is a scientifically objective methodology. 


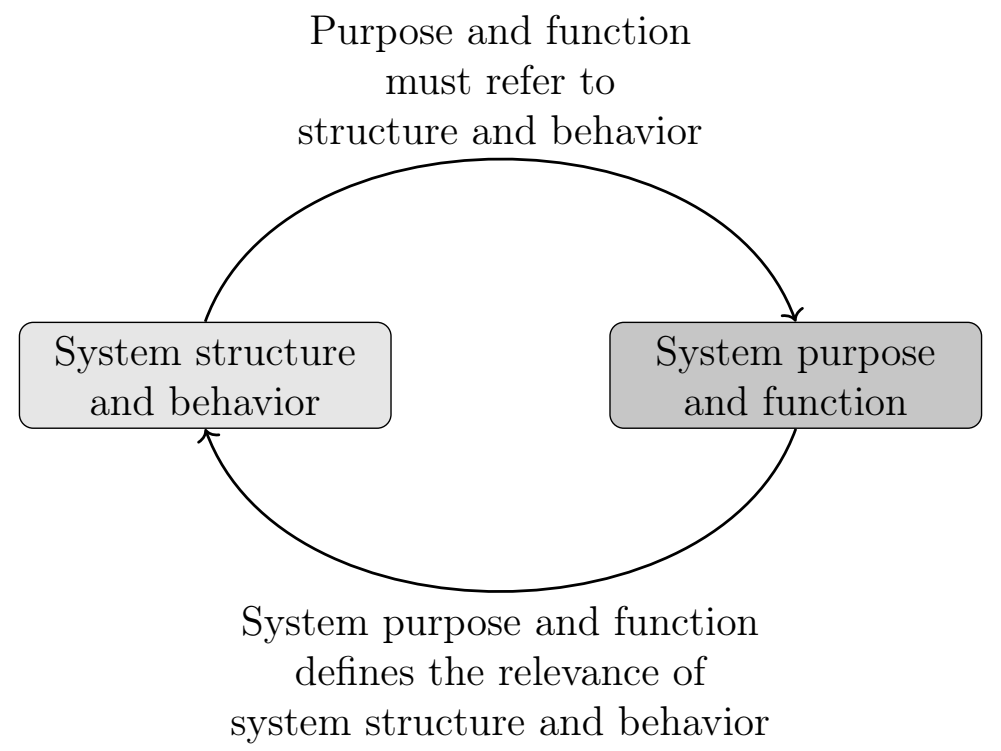

Figure 1: Modelling hermeneutics. The double articulation of purposes. On one hand the system purpose and function depends on the system structure, phenomena and behaviour for the problem under investigation. On the other hand the system purpose and function define the relevance of the system structure, phenomena and behaviour. The system purpose also defines the purpose of the functional model.

Wimsatt ${ }^{13}$ proposes a general symbolic form for 'teleological' function statements:

$$
F[B(i), S, E, P, T]=C
$$

This equation states that according to theory $T$, a function of behaviour $B$ of item $i$ (or vice versa) in system $S$ in environment $E$ relative to purpose $P$ is to do $C$. It is important to indicate the dependence of $\mathrm{B}(\mathrm{i}), \mathrm{S}$, and $\mathrm{E}$ on $P$ and $T$. Wimsatt ${ }^{13}$ indicates that to the extent that the above three senses of function (i.e. perspectival, consequential and teleological) do fit equation 1 then derivative functions also will fit the schema. Functional statements are convenient in cases where the perspective is such that either evaluative standards are being used or teleological explanations are appropriate.

The logic argumentation between the system purpose and the purpose of its symbolic representation, the functional model purpose, is illustrated in Figure 2, as a communication between the system (object) properties and functions aimed at fulfilling the system purpose 


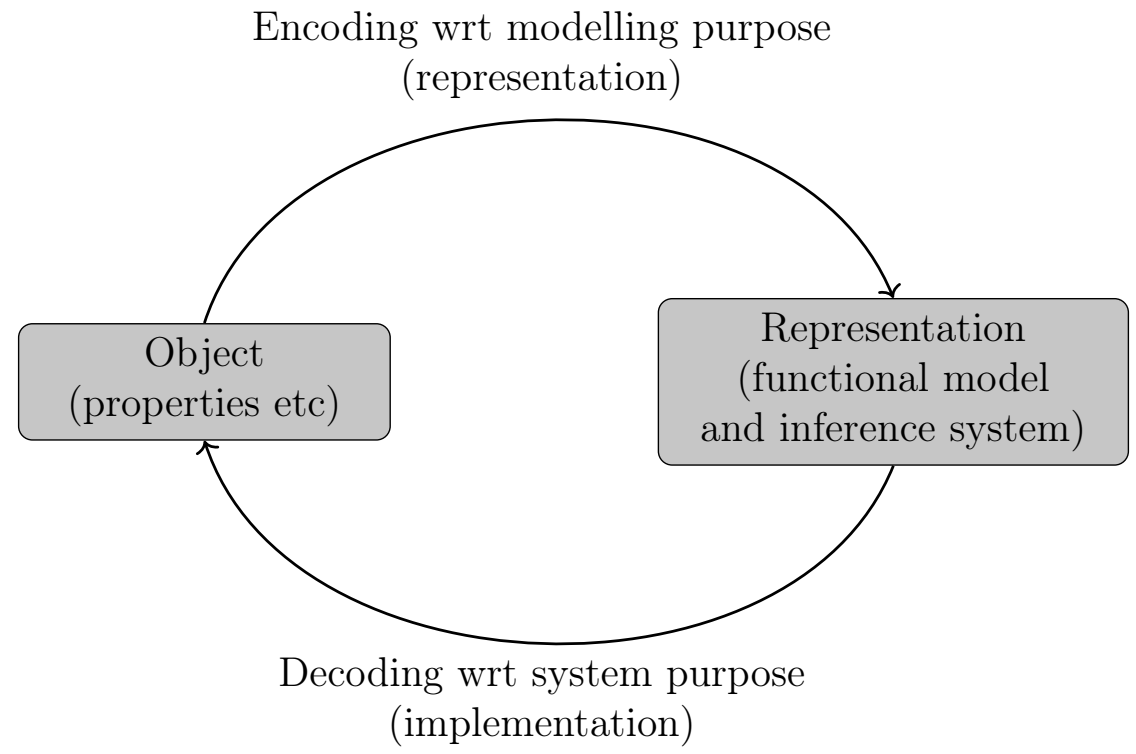

Figure 2: Illustration of the logic argumentation between the system with its purpose and the purpose of the symbolic representation, i.e. the functional model purpose.

and the symbolic representation of the system and its ability to represent the system purpose. The workflow through a design cycle is that once the system purpose is roughly defined a functional model aimed at representing the system purpose is developed. Based upon this first representation a first version of the system may be designed. If the proposed system performance compared to the system purpose is not satisfactory then the system purpose or the functional model may be modified and a new iteration may be undertaken. Clearly the better the system purpose can be defined a priori the faster convergence can be achieved. If the system purpose can be represented sufficiently well by the purpose of the functional model, then reverse engineering is directly applicable and iterations may be avoided. The en- and de-codings are further detailed in Figure 3 illustrating the logic argumentation taking place in the top rhombus symbol. At the top rhombus the overall system goal is translated into desired system purpose, e.g. represented by properties, as the first step of the modelling hermeneutics. Subsequently the system functionality is modelled based upon available theory to represent these properties. At the bottom circle the decoding of the symbolic representation provides a functional model representation of the purpose of the 
system. That representation can conveniently form the basis for a reverse engineering design of the system. It is interesting to point out that when a near final design is available then it may be validated experimentally; such comparison also will take place in the top rhombus. However validation should include evaluation of both the system purpose and the system performance. In summary the central functional modelling formulation issue is proposed to be called functional modelling hermeneutics, defined as follows:

Functional Modelling Hermeneutics: Translating the overall modelling goal into desired system properties, identifying the needed theory to model the system to represent these properties and identifying criteria for the evaluation of system purpose and performance.

Consequently, it is clear that functional modelling for the purpose or object (note that object is here used in the sense of what is aimed at, i.e. that towards which the mind is directed at in its activity) under investigation which forms an essential basis for product and process systems engineering as illustrated in Figure 3.

Function constitutes a concept between the intention of the designer or user and the behaviour of the artifact. In general there is no clear definition of this concept and it seems impossible to describe function objectively according to Umeda and Tomiyama. ${ }^{15}$ However, the functional modelling concept for process and product systems engineering presented above aims at developing a model of a system from a teleological point of view. ${ }^{16}$ The functional modelling concept describes how a system goal is translated into system properties, the properties are modelled using available theory (in so far as possible) and subsequently the obtained functional model is used for design, e.g. reverse engineering design. Consequently the overall goal is translated into desired functionality which then is represented through modelling using available theory and subsequently the model is used for design. The performance of the obtained design is compared to the system goal, if satisfactory then fine. If not, then either the original system goal, the translation of the goal into properties, the modelling or the design procedure may be modified to improve the performance. ${ }^{17}$ 


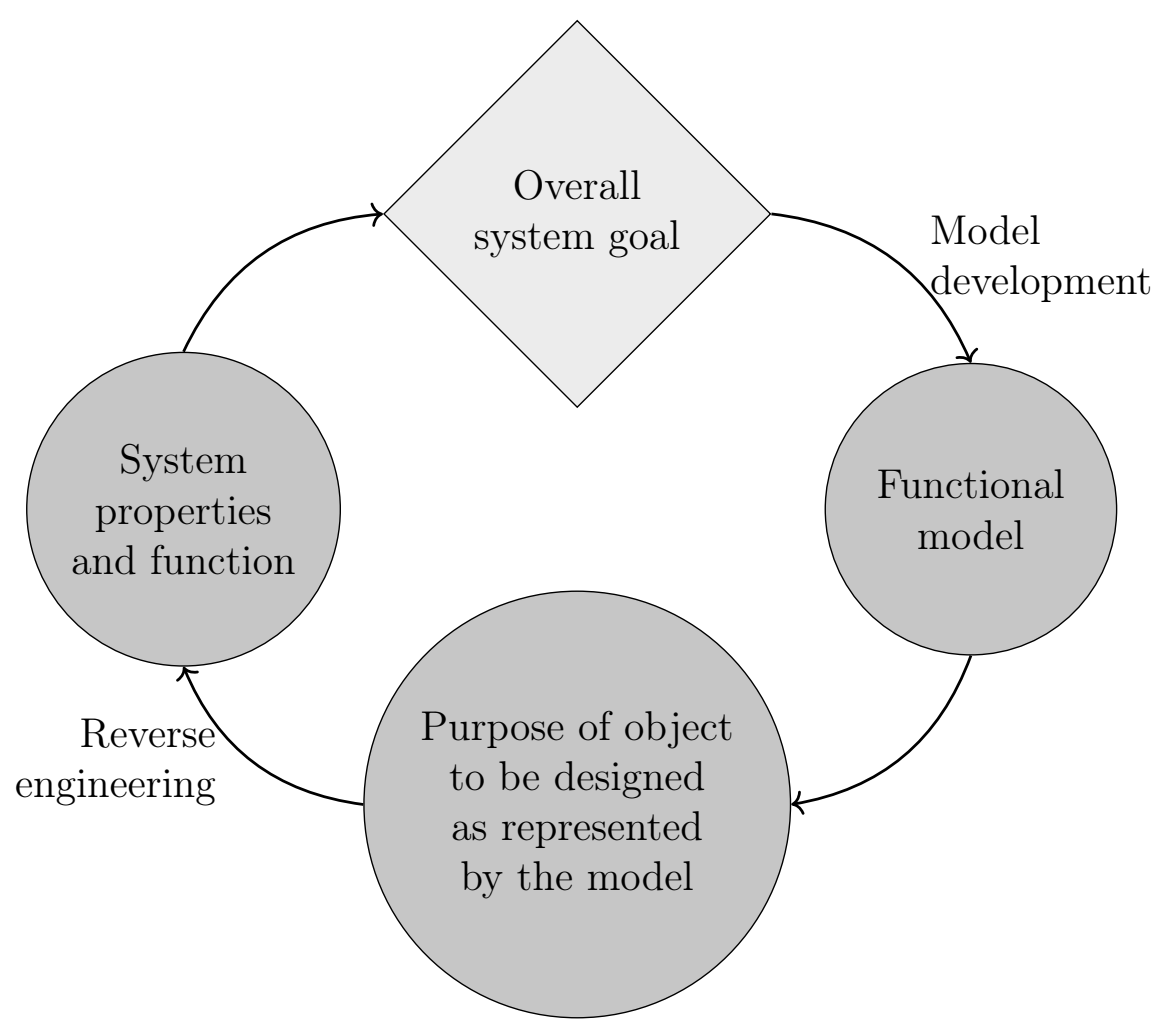

Figure 3: Illustration of the logic argumentation between the overall system goal and the purpose of the system to be designed as represented by the functional model.

As an illustrative example consider the following: One of the challenges in the operation of an oil and gas platform in some ocean is to balance the operation of the platform such that oil and gas streams flow continuously while the platform receives mixtures from several wells. To design a monitoring system for the purpose of balancing the operation a model is to be developed. The purpose of the model is clearly: To balance the platform operation. In order to fulfil this purpose a model which can support monitoring the mass flow of gas and liquid through the platform is to be developed. This example is pursued in the following subsections initially for the purpose of monitoring. Later also modelling for design and for dynamic simulation are briefly mentioned, and finally also causal and consequence reasoning are illustrated..

Below the development of different types of functional process models are described. First 
development of different functional process models is introduced. Thereafter development of well known quantitative computational physicochemical process models is presented.

\section{Qualitative Functional Process Models}

Following functional modelling hermeneutics, then functional modelling in process systems engineering is fundamentally based upon action theory, i.e. to combine the necessary theory and physicochemical properties with a physical topology to represent the desired system purpose, as illustrated in the left half of Figure 4. Qualitative functional modelling for the purpose of the object under investigation involves development of a symbolic representation using means-end concepts, such that, in a two dimensional view, the process causal interactions and behaviour are described with part-whole concepts in one dimension to achieve the system purpose, which is represented in the means-end dimension as illustrated in Figure 5.

The means-end dimension provides a conceptual distinction between the plant objectives, the functions provided to achieve the objectives and the physical structure realizing the functions. This dimension accordingly represents the causal factors and relations (structure and functions) whose intended effects support the achievement of objectives. Thus the basic principles of means-end analysis are utilized for the identification of relations between plant objectives and the causal factors i.e. the plant physical structure and its functions. The part-whole dimension is used to decompose objectives, functions or the physical structure into parts, thereby enabling representation of a system on several interconnected levels of abstraction.

For a particular system purpose a model is developed where the necessary detail, i.e. the granularity, depends upon the system purpose. For a process model with qualitative granularity this would include the first engineering principles balances for the relevant phases with the relevant phenomena and qualitative properties in the part-whole dimension and qualitative information about contribution to fulfillment of the represented system purpose including control in the means-end dimension. Thus a qualitative functional model can 


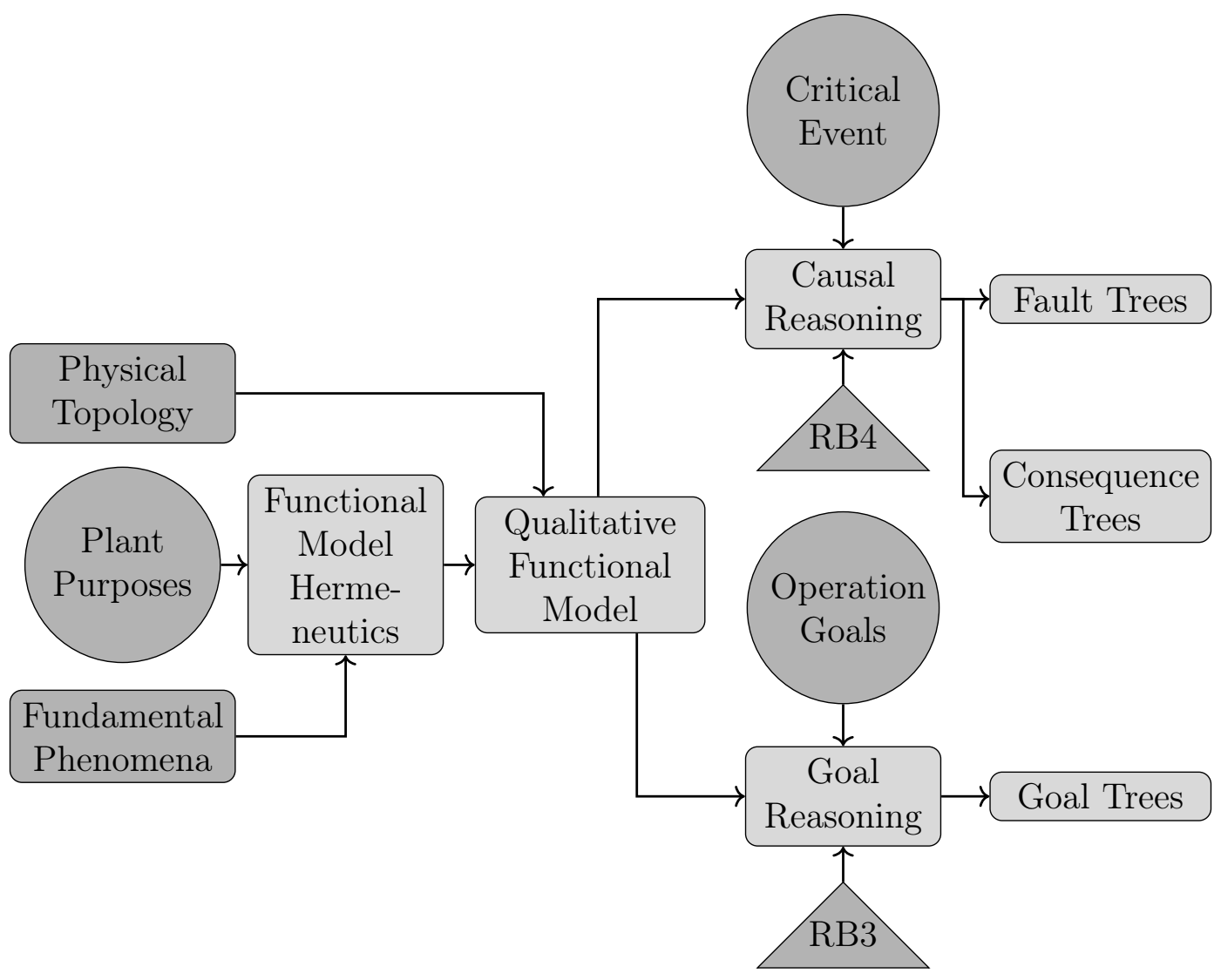

Figure 4: Qualitative functional model development including phenomena, causality and structure in the part-whole dimension and information related to fulfillment of system purpose in the means-end dimension. The right half of this Figure represents the causal reasoning where RB is a rule base used for backward and forward causality reasoning to develop fault and consequence trees respectively.

be developed which represent causal interactions between flows of material and energy, the equipment and the control systems as well as relations to evaluate the fulfillment of the represented system purpose. A qualitative functional model represents the objectives and functions of complex systems with different relation types in the means-end and part-whole dimensions. Therefore a qualitative functional process model can be used to analyze dependency relations between different functions and objectives. Consequently reasoning can be based on generic cause-effect relations when well defined functions and relations are used for developing the qualitative functional model. ${ }^{18,19}$ Such a knowledge base is efficiently build for reasoning within a model based expert system. ${ }^{20}$ 


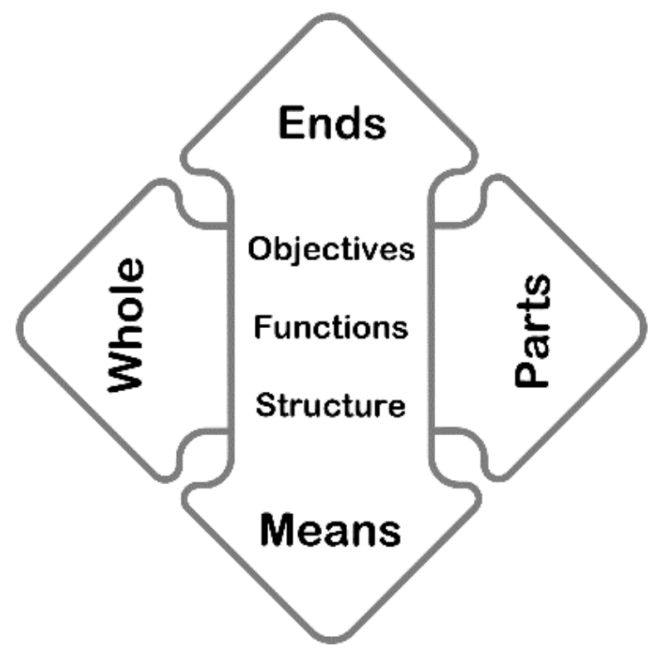

Figure 5: Illustration of the Means-end and part-whole dimensions of qualitative functional modelling for the purpose of the object under investigation. Note that in a top down view the end is supported by the objectives which are achieved by the functions which are realized by the physically structured implementation of the means

A qualitative functional model may be used early during process plant synthesis or during plant operation for monitoring process operation, e.g. for alarm handling by developing other functional model representations, as illustrated below. When a critical event occurs fault diagnosis may be performed since a fault tree can be developed through backwards reasoning. Similarly for evaluation of the consequences of a fault, this may be carried out since a consequence tree may be evaluated through forward reasoning. These possibilities are both illustrated in the right hand half of Figure 4. The fault and consequence trees are developed by a reasoning tool with a rule base for the causal behaviour of the represented phenomena. This is in contrast to traditional fault tree analysis and event tree analysis where the trees both mostly are developed manually. The above mentioned functional model based trees are obtained by a qualitative reasoning system through a formalized modelling language Multilevel Flow Modelling (MFM) developed by Lind and coworkers. ${ }^{21}$ These aspects are further elaborated under process monitoring in the applications section.

Addressing the example of balancing the operation of an oil and gas platform, then the modelling obviously must be based upon first engineering principles 
for mass and energy. However, a qualitative model representation should suffice for the balancing purpose where the model enables following the state of the units on the platform relative to normal operation, e.g. by using a three level granularity of the states with low, normal and high. Such a model receives the streams of oil/gas mixtures from several wells in a manifold, this mixed stream flows into a high pressure three phase flash separator where gas, oil and water are separated, subsequently the gas stream flows into a lower pressure separator where from the gas stream is dried in a dehumidifier and finally is compressed and lead to a gas pipeline for transport to shore. The oil and water streams are further separated and the oil is compressed and sent to an oil pipeline to shore, while the water stream is rinsed through further separation in a hydrocyclone before it is compressed and reinjected into a suitable well. Such a qualitative functional model of the oil and gas platform could be most useful for monitoring the operation of the platform, but also for reasoning about critical events, e.g. the consequences of the loss of one of the wells and for building fault trees in case of a critical event. However the qualitative modelling environment must enable such reasoning.

\section{Quantitative Physicochemical Modelling}

In traditional development of a physicochemical model the first step includes the conceptual mental activity of the modeller to decide which phases, balances and pathways to represent in the model in order to be able to evaluate the performance of the system. ${ }^{1}$ This first step corresponds to the functional modelling hermeneutics defined above for the purpose of the object under investigation and illustrated in the left hand half of Figures 4 and 6. For a process model with quantitative state granularity the modeller in subsequent steps must include property models, e.g. through the means of data bases and a rule base to select a suitable model for the relevant molecular and transport properties as well as a physical 
topology. Subsequently the modeller obtains the algebraic and/or (partial) differential equations together with initial and boundary conditions and estimated parameters to represent the system behaviour and constraints as illustrated in Figure 6.

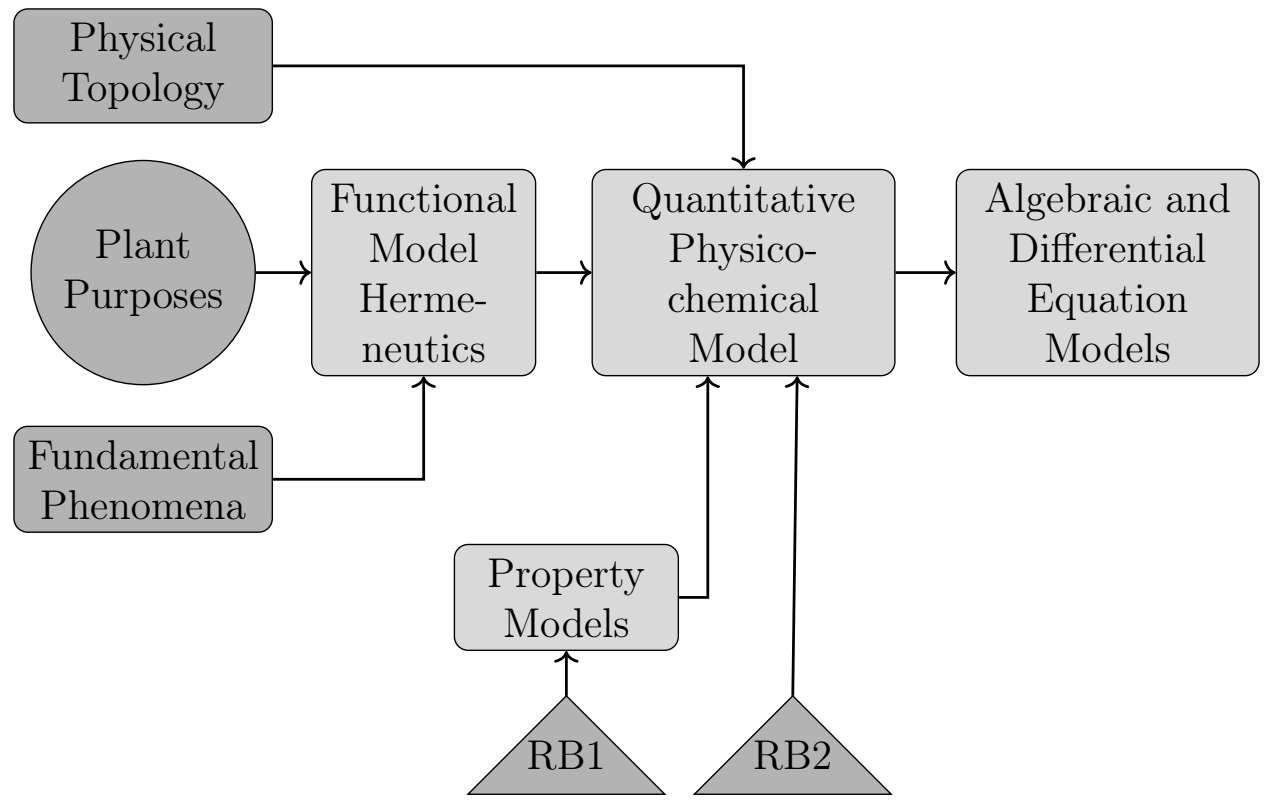

Figure 6: Qualitative functional Model development based on fundamental phenomena and structure in the part-whole dimension with information related to fulfillment of plant purpose in the means-end dimension (left hand side of figure). Quantitative physicochemical model development based on the same functional model hermeneutics and first engineering principles but now with quantitative state representation including rule bases (RB) for properties and for quantitative model synthesis (right hand side of figure).

Returning to the oil and gas platform example, but here for the purpose of designing the platform a quantitative physicochemical model can be developed from the functional model. This is achieved, as illustrated in Figure 6 by selecting real numbers to represent the states of the components, by selecting a suitable thermodynamic model to represent the behaviour of the oil and gas equilibrium properties in the first principles mass and energy balances for the unit operations on the platform.

Thereby the quantitative physicochemical model also is based on functional model hermeneutics developed for the purpose of the object under investigation. This computational quan- 
titative physicochemical model can be used to design the platform through repeated static simulation, e.g. using mathematical optimization, through focused functional modelling of e.g maximal driving force or a combination thereof. If instead dynamic simulation is the purpose then a dynamic version of the quantitative physicochemical model is developed and solved using a suitable dynamic solver.

Quantitative mathematical models are routinely and efficiently simulated for design and analysis of static and dynamic behaviour of the system under study. Many different environments are available for modelling purposes, e.g. MODEL.LA ${ }^{22}$, OMOLA $^{23}$, ASCEND ${ }^{24}$, Modeller ${ }^{25-27}$ and ICAS. ${ }^{28}$ Several commercial static and dyamic simulation packages with rigorous quantitative models are available and widely used, e.g. ASPEN, ${ }^{29} \mathrm{HYSYS},{ }^{29} \mathrm{PRO} / \mathrm{II}$ and gPROMS. ${ }^{30}$ Such tools can be effectively used for analysis of system performance and for validation of system design. These tools are also very efficient for evaluation of controlled system performance through dynamic simulation.

Quantitative physicochemical models can support system analysis and design through iterative solution, e.g. by optimization or through focused functional modelling, e.g. based on maximizing driving force. However the key point in the context of functional concepts is that physicochemical mathematical models generally do not support reasoning directly only indirectly through iterations. For quantitative physicochemical models system performance is evaluated based upon detailed static or dynamic system simulation or possibly a set of simulations rather than upon qualitative reasoning relative to fulfilment of plant purposes which is possible in qualitative functional models. Consequently the two model types are complementary. The difference between qualitative functional models and quantitative physicochemical models illustrates that the qualitative functional models can be extremely useful for reasoning about fulfilment of the model purpose at the conceptual level, while the quantitative physicochemical models can be used for detailed simulation both for analysis and design and for dynamic simulation relative to satisfying operational performance specifications. 
In the following section selected application areas of functional modelling concepts are reviewed briefly. The emphasis is on the underlying conceptual phenomenological theory and the modelling hermeneutics, i.e. the translation of the system goal to model properties that can be represented by modelling the phenomenological theory in the different application areas.

\section{Application of Functional Concepts}

To model the function of chemical products and processes it is necessary to model the properties of chemicals. Therefore the development of mainly one class of models for chemical properties is presented before reviewing design of chemical products. Thereafter synthesis, design and innovation of processes is reviewed before selected developments in process control and monitoring are presented. It is important to note that concepts and formulation are central in this paper, but otherwise solution strategies are not covered.

\section{Chemical Product Property Modelling}

The properties of chemical molecules obviously play an important role both for the functionality of chemical products themselves and for the function and behaviour of molecules in process plants. For simple qualitative models the behaviour of the chemical products may be described using simply state of matter, i.e. gas, liquid or solid, however only simple conceptual phenomena may be described. For quantitative models modelling the properties of chemical molecules is crucially important for both product and process systems engineering. Pure component and mixture properties depend on temperature, pressure, composition and molecular structure. Accordingly a classification of properties is proposed ${ }^{31,32}$ as primary and secondary properties. Primary properties are those that depend on the structural, chemical or physical parameters related to the molecule and/or mixture only. Secondary properties are those that in addition to the parameters of the primary properties also may be functions 
of other primary or secondary properties.

Property models have been developed to predict molecular properties from structural descriptors. Property models can be classified into mechanical models, semi-empirical models and empirical models. Most practitioners have relied on semi-empirical quantitative structure property relationships (QSPRs) to provide a clear connection between the structural chemical space and the space of quantitative functional properties. These methods are also often simple and can be applied to estimate properties very efficiently. ${ }^{33}$ Most QSPR methods break molecular structure into sub-molecular groups of atoms and bonds. These molecular sub-structures are assumed to dictate molecular properties. An important class of semiempirical property models is the group contribution (GC) models, where the properties of a molecule are determined by adding the occurrence frequency times the contribution of each molecular group in a molecule. Such first-order property methods consider molecular groups to be non-overlapping and independent of the structure of the molecule. However, proximity effects such as interactions between molecular groups are not accounted for. Constantinou and Gani ${ }^{32}$ and Morejon and Gani ${ }^{34}$ introduced second and third order molecular groups which have the first order groups as building blocks. The higher order groups are able to capture interactions between molecular groups, such that isomers can be distinguished and properties of poly-functional molecular groups can be estimated.

Other property models apply topological and atom Connectivity Indices (CI), which are structural descriptors calculated based on chemical graph theoretical principles. Since experimental data for the required molecular group to represent a chemical compound is not always available in the GC methods, Gani et al. ${ }^{35}$ presented a combined GC-CI method called GC+. With this method molecular groups which are unavailable in GC methods are predicted through CI. Hukkerikar et al. ${ }^{36}$ improved the reliability of the GC+ method by reestimating the pure component parameters and also a large number of environment related properties. The GC+ models provide property prediction for different chemicals such as pure compounds, mixtures, polymers and recently also amino acids. ${ }^{37}$ The ability to model these 
properties and their uncertainty enable synthesis, design, and analysis of chemical products and processes giving useful insights into the quality and reliability of the design of sustainable processes. The application of QSPR techniques to predict properties from structures, defines what is known as the 'forward problem,' which is what QSPR techniques are generally intended for. This ability to model the properties of chemical compounds also provide a basis for design of molecules for a specific purpose, using reverse engineering as illustrated in Figure 3. Computer Aided Molecular Design (CAMD) considers the 'reverse problem', that is the problem of predicting structure from desired properties. In the following focus will be on application of group contribution methods for product design.

\section{Chemical Product Design and Formulation}

Chemical product design involves according to Cussler and Moggridge, ${ }^{38}$ a procedure for defining what we need, generating ideas to meet this need, screening and selecting the best of the ideas and finally, deciding what the product should look like and how it should be manufactured. A chemical product can be defined as any chemical entity that enables a device or process to perform a desired function. The product and its properties are, therefore, deeply intertwined with its function. ${ }^{39}$ Chemical product-function pairs include, e.g.: solvents to enable industrial separations, refrigerants to enable heat pumping, additives to increase shelf life of foodstuff or to increase appearance of human body in cosmetics, pharmaceuticals to fight microbial infections, fuels to convert chemical energy to mechanical work, lubricants to facilitate energy transfer and many more. The chemical products may be delivered as single species products (solvents, refrigerants, or active ingredients), multiplespecies products (blends, lubricants), formulated products (cosmetics, cream, detergents) and devices (air filters, humidifiers, controlled release capsules for delivering chemicals for a purpose). Clearly the more general case of chemical product design requires a mix of skills from different disciplines such as business, social sciences, and fine arts, not only chemical

sciences and chemical engineering. ${ }^{38,40}$ Consequently, chemical product design involves a 
multi-stage procedure of identifying the best set of candidates that fit desired functional product criteria. Experimental trial-and-error design involving generating new formulations, guided by previous products or expected (predicted) performance of new ingredients, and testing these formulations against design criteria. The search space that can be practically investigated using trial-and-error methods is severely limited by the effort, cost, and time required. In a functionality based process/product design paradigm multiple stages are needed where each stage ideally needs a functional model representation to support the desired purpose, i.e. the functionality at that stage. However many functional aspects in product design cannot yet be modelled based on fundamental phenomena, Therefore in this paper the focus is on design of product molecules with desired functionality and on blends, where it is possible to develop methods that combine property predictive models with computer-assisted search. ${ }^{39}$ The focus is especially on CAMD with GC based property modelling.

Computer Aided Molecular Design (CAMD) refers (as mentioned above) to the design of molecules with desirable properties, i.e. a certain value of thermodynamic properties such as melting point, boiling point, surface tension, and viscosity; or implicit properties, e.g. heat transfer capacity of refrigerants or aroma of fragrances and taste. Through CAMD, one determines molecules that match a specified set of properties. CAMD as a technique has a very large potential as in principle, all kinds of chemical, bio-chemical, and material products can be designed through this technique. It has become a mature technique and is attracting more and more researchers and finding increasing industrial applications. ${ }^{41,42}$ 'Generate-and-test' synthesize design methods were developed by Gani and Brignole ${ }^{43}$ and Joback and Stephanopoulos ${ }^{44}$, based on rules to generate molecular structures by combining functional groups and use of the same functional groups for test of the molecular structures with GC-based property estimation techniques. In these qualitative knowledge based techniques the product design problems are solved indirectly by generating alternatives and testing them for feasibility, i.e. through a systematic reduction of the number of alter- 
natives through heuristics ${ }^{45}$ where a set of rules is derived from a combination of experience, insights, and available knowledge. A major difficulty with the rule-based methods is that the rules are sometimes contradictory and therefore difficult to apply, and only valid within a narrow product context. Heuristic design methods for cosmetic products like creams and pastes, were proposed by Wiboro and $\mathrm{Ng} .{ }^{46}$ Harper and Gani ${ }^{47}$ proposed a multistep and multilevel version of the 'generate-and-test' method for CAMD, The objective of the problem formulation algorithm is to transform the qualitative goal specification into a quantitative specification of the desirable properties and their ranges through a combination of the use of knowledge base, insights and experience. It is a multi-step procedure requiring different levels of information. As an example the synthesis of molecules or mixtures to satisfy the functional goal is developed in three phases. ${ }^{48}$ In the predesign phase the desired goal, i.e. the qualitative functional behaviour of the molecule is matched with the properties of the final molecule. The range of the goal values of these properties with respect to the desired functionality is determined. The methods for determining the properties are identified. And finally the building blocks for generation of molecular structures are selected such that undesired molecular blocks are screened out. In the design phase a set of feasible candidates is generated and tested against functional properties through a multilevel procedure to prevent combinatorial explosion. In the post-design phase the candidate molecules are ranked according to the extent to which they satisfy the desired behaviour. Gani et al ${ }^{49}$ extended the multistep and multilevel CAMD method for selection and design of solvents for organic synthesis. This method was further extended by Satyanarayana et al. ${ }^{50}$ for design of polymer repeat unit based products, such as bottle stops, synthetic fabric, or a heat-resistant film. Solvason et al. ${ }^{51}$ developed a systematic method for CAMD through a graphical approach. Here, the solution method is composed of three steps: (1) determination of the feasible property target region, (2) identification of candidate additives and ingredients, and (3) product validation. The graphical representation is based on the property cluster method. ${ }^{52}$ Through the mathematical programming technique the product design problems are solved 
directly through an appropriate numerical solver as reviewed by Austin et al. ${ }^{33}$ A wide range of numerical methods have been developed and applied to solve the various design problems. A selection thereof are highlighted here. A major difficulty for mathematical programming techniques is the size and complexity of the mathematical programming model. The scope and significance of the problems depends on the availability and application range of the models used. Odele and Macchietto ${ }^{53}$ used mathematical programming in chemical product design for the optimal solvent selection problem. Venkatasubramanian et al ${ }^{54}$ applied genetic algorithms to solve CAMD problems. The application of mathematical programming methodology was expanded to polymer design ${ }^{55}$ and refrigerant design with the inclusion of environmental impacts as constraints. ${ }^{56}$ Maranas $^{57}$ employed MINLP models for molecular design with uncertainty in the functional group parameters. Raman and Maranas ${ }^{58}$ and then Camarda and Maranas ${ }^{59}$ proposed the use of connectivity indices for representation of the molecular structures and employed MILP models for chemical product design. Sahinidis et al. ${ }^{60}$ also used an MINLP model, but for a refrigerant design problem. Sinha et al. ${ }^{61}$ extended the MINLP models to the design of solvent blends and employed interval analysis to obtain the optimal blend. However, this type of methods can be computationally expensive for large problems. This issued was addressed by Karunanithi et al. ${ }^{62,63}$

Hybrids of the rule based and mathematical programming methods have been used to develop efficient methods for design of blended products ${ }^{64}$ where also life cycle assessment methods have been included. Such tools are finding increasing industrial use because of their potential to quickly generate and evaluate thousands of candidate products; to estimate a large number of the needed physicochemical properties for selecting a small number of feasible product candidates for further refinement through experimental verification. For such tools Computer Aided Mixture/blend Design $\left(\mathrm{CAM}^{b} \mathrm{D}\right)$ was defined as follows:

Computer Aided Mixture/blend Design: Given a set of chemicals and a set of property constraints, determine the optimal mixture and/or blend consisting of a sub-set of chemicals. ${ }^{64,65}$ 
The design of chemical products depends very much on the ability to represent the product characteristics by a set of measurable and/or predictable properties. It is noteworthy that much work is required a priory to obtain such properties. For example, it is necessary to gather information from consumers in terms of basic needs for defining the main functions of the products, and additional needs, which would improve the product quality, as well as know-how to translate the consumer needs into target properties. Usually, an expert would be able to provide this knowledge if the necessary data is available. Another option followed in Kalakul et al. ${ }^{64}$ is to collect necessary data from published expert insights, open source databases, patents, published articles, etc., to prepare the translation of needs to properties in the same way as Conte et al. ${ }^{66}$ used for liquid formulated products and others used for single species chemical products for CAMD. ${ }^{41}$ It should be noted that this is a time consuming work, but availability of such data, avoids this extra work. For a new type of chemical product not covered by similar information in the product design software tool, extra work would be needed to establish this translation. From a functional view the central problem formulation issue in product design can be called product design hermeneutics, defined as follows:

Product Design Hermeneutics: Formulating an overall product design goal with additional needs. Translating the product design goal and additional needs into a set of sub-goals, i.e. target properties where each sub-goal can form the basis for a task in the design procedure. Identifying the needed theoretical bases, if available, to represent the target properties and identifying the evaluation criteria for a product design.

Product design is clearly a functionality driven discipline since the product design goal to a very large extend is determined by the intended purpose of the product. 


\section{Process Design and Innovation}

Traditionally chemicals-based products and their corresponding production processes have been designed and developed through heuristics-based experimental approaches, where scaleup is a major issue ${ }^{67}$. Although this approach often leads to the development of a safe and reliable product and a corresponding production process, it is not practically feasible to evaluate all alternatives with this approach. That means that a better product and/or a more efficient process may have been missed. Use of model-based computer-aided tools provides an alternative to the experimental based approach. With the advances in computer technologies, it is now possible to routinely study various aspects of, e.g. chemical process design, operation, and control with model-based computer-aided methods and tools. For example, the use of a process simulator to perform mass and energy balance calculations as part of process design and optimization is well-established.

Taking a look at the traditional design of a product for a specific purpose then first the chemical identity of the product and its formulation has to be determined. Then possible routes for the production of this product from the most feasible available raw materials have to be identified before the more promising production processes and topology with the necessary process operations can be synthesized. In such a production plant there may also be a need for special aiding components such as solvents and membranes that have to be selected, or even designed for the specific purpose. Finally the most promising production plant can be designed and constructed while the operation of the plant is planned. Throughout this design development procedure the aspects of sustainability, safety and control have to be addressed as well as economy. ${ }^{68}$

A traditional process plant development procedure involves a number of tasks, such as product design, process design, scale up, control design, formulation of operational procedures, monitoring, production planning, etc.. Each of these tasks may be considered as a set of specific sub-tasks, where different aspects of product/process modelling may be effectively utilized to provide a basis for resolving the task. To the extent that the sub-goal of the 
particular stage during the process development can be modelled, then each stage may represent one cycle in the logic argumentation between the system sub-goal and its symbolic representation as illustrated in Figure 3. Obtaining a satisfactory resolution for each subgoal is strongly dependent on other tasks as the product/plant design develops and on the overall efficiency and environmental constraints. Thus the product/process design requires an overall approach. To represent the sub-goal at each stage often requires different symbolic representations. Furthermore the plant-wide considerations related to energy and material efficiency, process safety and environmental aspects also require different symbolic representations. Consequently, a set of different models and associated tools are needed for flexible product/plant development. Since the models vary also the reasoning tools to support decision making at each stage of the process development will vary. The interpretation of the overall product/plant goal into a set of subgoals may be viewed as as logic argumentation between the overall goal and the subgoals. Just as each subgoal, where modelling is relevant is involved in its modelling hermeneutics then also the overall product/plant goal will be involved in its design hermeneutics. Design hermeneutics involve first the translation of an overall product/process design goal into a set of sub-goals where each sub-goal can form the basis for a task in the design procedure. The further steps include identification of the needed theoretical bases, if available, for symbolic representation of the sub-goal property/-ies as well as identification of the relevant evaluation criteria.

Computer-aided methods for process synthesis and intensification may be classified as: heuristic, mathematical programming and hybrid. ${ }^{5}$ As mentioned above the earliest were heuristic methods which are rule-based where a flowsheet is selected through the application of qualitative process-insight based rules at the unit operation scale and know-how. Siirola, Powers and Ruud ${ }^{69}$ proposed a hierarchical task-based approach for screening unit operations, selection and combination of individual tasks for reaction schemes. Douglas ${ }^{70}$ proposed a hierarchical rule-based decision procedure for selection of the reaction and separation structure of a feasible flowsheet alternative. Mathematical programming methods are 
optimization-based methods/algorithms that are used to select a flowsheet from a superstructure of alternatives by evaluating all alternatives using an objective function. These methods have been developed by Grossmann and coworkers, and recently reviewed by Chen and Grossmann $^{3}$ and Cabeza et al. ${ }^{71}$ Raman and Grossmann ${ }^{72}$ showed how qualitative knowledge may be used systematically in mathematically logical expressions. Friedler et al. ${ }^{73}$ introduced the process graph framework named P-graphs to enable unique representation of feasible process flowsheets and provided an algorithm for determining the set of all feasible flowsheets thereby reducing the optimization effort. Ierapetritou et al. ${ }^{74}$ handle uncertainty through a stochastic optimization procedure. Hybrid methods are based on combining information from phenomena based heuristic and mathematical programming methods. Predictive models are used so far as possible to provide sufficient physical insights to reduce the network of possible flowsheet alternatives. Thus, the synthesis problem involves fewer, but feasible alternatives for evaluation than mathematical programming. Jaksland et al. ${ }^{31}$ proposed a method based on thermodynamic and physical insights from the analysis of pure compound and mixture properties for sequencing of separation tasks. Karunanithi et al. ${ }^{62}$ proposed a decomposition-based, solvent-process synthesis method for the design of crystallization processes. Hostrup et al. ${ }^{75}$ proposed a product-process synthesis methodology for integrated solvent selection using CAMD and process design for separation of azeotropes. Gani and Bek-Pedersen ${ }^{76,77}$ proposed a driving force-based graphical method for the optimal sequencing of distillation columns requiring minimal energy conversion. d'Anterroches and Gani ${ }^{78}$ proposed a process group contribution based approach to solve the synthesis-design problem of chemical processes, where, chemical process flowsheets could be synthesized in the same way as atoms or groups of atoms are synthesized to form molecules as outlined above through the computer aided molecular design technique. The process groups are defined using e.g. the driving force for distillation. The parameters for the process groups are obtained through simulation of rigorous models. This phenomena based synthesis methodology is clearly inspired by the computer-aided molecular design method CAMD. ${ }^{43,47,67}$ The methodology has 
been further developed by ${ }^{79}$ introducing functional process-groups (building blocks) representing a wide range of process operations, and defining a set of flowsheet connectivity rules to join the process-groups into all feasible flowsheet alternatives and flowsheet property models. Thereby they determine energy consumption, atom efficiency, environmental impact to evaluate the performance of the flowsheet alternatives.

To eliminate the limitation of unit operations and achieve even larger material, energy and economic benefits Process Intensification (PI) has been introduced. This field is reviewed by Greven and Stankiewicz ${ }^{80}$ and more recently by Sitter et al. ${ }^{81}$ The former authors review chemical process integration from the molecular to meso- and macroscale. Consequently process integration may be defined as the improvement of a process through the targeted enhancement of performance-limiting phenomena at different scales. ${ }^{82}$ To achieve such improvements it is clear from a functional point of view that process synthesis and design must be investigated at a lower level of aggregation, i.e. below the task level. ${ }^{83,84}$ For that purpose Papalexandri and Pistikopoulos ${ }^{83}$ proposed a generalized modular representation framework for process synthesis, Siirola ${ }^{85}$ proposed a phenomena based means-end analysis at the task level, and Pechel et al. ${ }^{86}$ presented an approach based on elementary process functions derived from functional modules to synthesize novel reactor networks. Rong et al. ${ }^{87}$ proposed a process synthesis based on manipulation and variation of process phenomena while Arizmendi-Sanchez and Sharratt ${ }^{88}$ proposed a set of typical functional phenomena based building blocks as a basis for qualitative modelling to support reasoning leading to process intensification using quantitative modelling. Lutze et al. ${ }^{89}$ proposed a systematic multi-step approach towards phenomena-based process synthesis, building on an identification of necessary and desirable phenomena, as well as the generation and screening of phenomena-based flowsheet options by means of a decomposition based solution approach. The phenomena based building blocks (PBB) are abstract functional physical elements (i.e. balance volumes) in which functional physicochemical phenomena, such as mixing, phase contact, phase transition, phase change, phase separation, reaction, energy and momentum 
transfer or stream division, take place. These PBB can be combined to form simultaneous phenomena building blocks (SPB) in the same way that atoms can be combined to form functional groups. Thus SPB can be combined to form basic process plant structures in the same way molecular groups can be combined to form molecules. These basic structures fulfill a task or set of tasks in a more sustainable manner. Therefore, they can be translated into existing or novel intensified unit operations in the same way as molecules developed by CAMD can be identified through a database search of existing molecules. If a unit operation cannot be found that matches the basic structure then, in principle a novel, intensified unit operation is generated, for example, a membrane assisted reactive distillation. ${ }^{89}$ This is analogous to CAMD because if a specific molecule cannot be found from a database search then a new molecule, i.e. one that satisfies the required designed properties, has been generated. From a library of building blocks (functional process-groups) and a set of rules to join them, chemical process flowsheets are generated and evaluated for properties such as energy efficiency, atom efficiency, safety factors, product recovery and purity, using functional process-group based property models. Environmental performance determined from sustainability metrics $^{90}$ and life cycle analysis (LCA) factors ${ }^{91}$ are introduced as intensification criteria. In this way, a list of feasible chemical process flowsheets is quickly generated, screened and selected for further analysis. In the next stage, the design parameters for process operation are established through reverse engineering approaches based on driving forces available for each operation. Also, new process groups are added to represent unit operations, e.g. for applications in bio-processes. In the final stage, when all the necessary information for a rigorous process simulation is available, rigorous simulation is performed to validate the synthesis-design. There are no iterations involved since the flowsheet is synthesized and the operations in the flowsheet designed to match a set of design goals. The final flowsheet is among the best, if not the best. This multi-level tool builds on the analysis of a base case design and has been further refined and extended towards the design of sustainable intensified processes by Babi et al. ${ }^{92}$ The framework involves a three stage 
work-flow where, in the synthesis stage an optimal processing route is synthesized through a network superstructure optimization approach with related synthesis tools. In the design stage, the processing route from the first stage is further developed and a base case design is established and analyzed. In the innovation stage, more sustainable innovative solutions are determined. The methods start with an analysis of a base case process design, which subsequently is transposed into a phenomena-based representation, to form the basis for the process intensification. The framework is presented as an integrated computer-aided tool. ${ }^{93}$ Evaluation of process intensification is important for ensuring its practical relevance. One of the process intensification metrics for evaluation combines four indicators: economic, environmental, safety and intrinsic intensified. ${ }^{82}$ Recently Rivas et al. ${ }^{94}$ has suggested an intensification factor to evaluate PI options. It combines qualitatively and quantitatively economic, technical, social and scientific aspects in a simple manner and enables evaluation from minimal information during early design.

Kuhlmann and Siborowski ${ }^{95}$ use an optimization based approach to handle the combinatorial complexity encountered when allowing for a generic combination of the PBBs and the numerical complexity that comes with the consideration of rigorous thermodynamic models in order to avoid previous simplifications that might render the final design infeasible. Demirel et al. ${ }^{96}$ proposes a set of fundamental building blocks that can be associated with different process phenomena, tasks and unit operations. Coupling of blocks is used to represent coupled phenomena where the boundary between such coupled blocks depends upon the particular coupling phenomna. Thereby a relative large set of possible blocks result. An assembly of blocks of the same type constitutes a classical unit, while an assembly of blocks with different types results in an intensified unit. These authors also report ${ }^{97}$ that a slightly extended optimization based methodology provides a single framework for sequential or simultaneous synthesis, integration and intensification of chemical processes.

The development of systematic tools for process synthesis, design and intensification clearly has been both directly and indirectly influenced by a functional view from the relatively 
early developments through the means-end analysis at the task level ${ }^{68}$ to the functional phenomena building blocks approach. These approaches have all facilitated the development of more sustainable processes. However the granularity of the phenomena building blocks differ and it is clear that with increasing computational power the phenomena building blocks may continue to increase the resolution of the fundamental phenomena. Consequently it may be expected that the hybrid approach may benefit from using the thermodynamic insight when selecting the phenomena building blocks.

\section{Integrated product and process design}

In a relatively simple case of chemical product design one main purpose is often to design a product, i.e. a commodity that enables driving a particular process. Thereby it is possible to outline the preferred product properties that provide optimum process performance without in advance deciding on any specific compounds. ${ }^{98}$ This problem setting constitutes an integrated chemical process and product design problem where the process target values are input to the product design. At the same time the product target values feed the development of the desired process, this case illustrates the logic argumentation depicted in Figure 3 which in this case represents integrated process/product development. To facilitate integrated process and product design, a virtual product-process design laboratory (virtual PPD-lab) software tool was developed based on a decomposition strategy. ${ }^{99}$ This tool is developed to tackle the design problem of complex products such as formulated liquid products, i.e. microstructures. In order to design this type of product, active ingredients, additives and solvents are combined such that the properties and functions of these complex mixtures are predicted. This integrated method includes the design/verification of formulated products as well as validation/testing of the final products. In a similar contex Scheffczyk et al. ${ }^{100}$ introduces a computer aided molecular process design framework $\left(\mathrm{CAM}^{P} \mathrm{D}\right)$ and demonstrates that the performance relies strongly on the available model to predict the molecular properties. Typically, approximate thermodynamic prediction methods such as GC methods are 
used, which assume the additivity of functional groups and require interaction parameters determined in experiments which limits the molecular design space. However, the presented framework is based on mechanistic, i.e. quantum-mechanics based thermodynamic property predictions and thus relies neither on experimental data nor on group additivity. As process models, the framework employs advanced pinch-based process models which assume infinite time for heat and mass transfer in separation unit operations which results in vanishing thermodynamic driving force at certain potentials, which are called pinch points. In a hybrid extraction-distillation case study, ${ }^{100}$ where the accurate pinch-based process models together with the mechanistic molecular modelling are able to account for the inherent trade-off in temperature dependent molecular properties between extraction and distillation, they demonstrate an experimentally validated $50 \%$ reduction in energy demand. However, the mechanistic methods are still computationally quite demanding.

Model based methods have progressed significantly through combined development of molecular modelling tools and application of qualitative functional knowledge in the form of know how or insights which enable goal directed development of model-based tools for solving many of the sub-problems in developing a product and process design. However it is also clear that the tools needed benefit from using models with varying granularity and from being based on the different limiting phenomena in a particular process, e.g. the pinch based models used by Scheffczyk et al. ${ }^{100}$ Thus a development of a new generation of tools which can facilitate such a flexible application of modelling is in high demand. ${ }^{5}$

\section{Plant Operation}

Plant operation depends crucially on process control and its structuring. Most often a simple basic control layer with PID and ratio controls are used to stabilize plant operation. For optimization of process plant productivity or economy then quantitative process models have been in use for many decades. Very early it was clear that the ideal control design for plants with no non-minimum phase characteristics simply was to invert the model, as 
exemplified by the inverse Nyquist array control design methodology; ${ }^{101}$ later developments enabled handling also the non-minimum phase parts as well, e.g. ${ }^{102}$. These control designs are simple examples of reverse engineering as illustrated in Figure 3. With the arrival of model predictive control (MPC) through industrial and academic contributions ${ }^{102-104}$ the application of both data driven modelling and first principles modelling have become widespread in industry for development of linear and nonlinear model predictive control. In the case where linear MPC with constraint handling is sufficient then process identification on the operating plants have become standard. ${ }^{103,105}$ Once a model is obtained to represent the desired performance under the given constraints, then a control system is designed to achieve the desired performance in the best way possible. With MPC this has become possible. The limitation then is the availability of measurement technology for key quality variables. When such are not directly available then estimation from secondary on-line or off-line measurements through soft sensors can become useful. ${ }^{106}$ Through Monte Carlo simulations the uncertainty of the necessary parameter estimation is feasible based upon suitably designed experiments. ${ }^{107}$ However, an important consideration is to use on-line filtering methods, e.g.various forms of Kalman filtering ${ }^{108}$ in order to combine on-line data with off-line data ${ }^{109}$ in a suitable manner. Often the MPC solution is used to develop a simpler but perhaps more robust solution based upon the stabilizing control layer with a few interactions with control of additional degrees of freedom. For more complex multivariable interactions with active constraints MPC provides an effective solution in cases where a first principles model or a data driven model is available. ${ }^{110,111}$ Clearly model development for control whether data-driven or first principles constitutes application of functional modelling. In data driven modelling significant aspects of qualitative knowledge is necessary, however the developed model has very limited predictive power.

Plant monitoring is critical for ensuring safety and process efficiency. Monitoring in industrial practice has been attempted by implementing alarm systems to provide safeguards to prevent the deterioration of near misses to accidents and to enable retrospective inves- 
tigation on a large number of accidents e.g. to collect accident data to find alarm system defects. Unfortunately industrial alarm systems suffer from mediocre performance since they often exhibit too many alarms which overload the operators, in particular because operator response time is often not explicitly taken into account when the individual alarms are designed. Clearly plant operators are presently not supported efficiently by existing industrial IT technology. ${ }^{112}$ Current supervisory control and data acquisition (SCADA) solutions are developed from engineering documents such as process and instrumentation diagrams (P\&ID), operating manuals and control logic under the assumption that these documents provides the required information for diagnosing deviations from normal operations and for planing the required emergency or mitigating actions. However, these documents are mainly developed for plant design documentation, which obviously is relevant for operations, but their information content is insufficient for planning operation actions. Supervision of operation is dependent on knowing the causal interactions between streams of material and energy, the equipment and the control systems, and how these causal interactions influence process behavior during disturbances as well as knowing the opportunities for intervention. Cause-effect relations can be derived from design principles for the plant and its subsystems and components. However, this knowledge is seldom documented in a form which is effective for operations. Decision making during disturbances is also dependent on knowledge of operating objectives both in the evaluation of consequences of disturbances, and in selecting among alternative courses of action. To ensure the reliability and safety of modern largescale industrial processes, data-driven and model based methods have received increasing attention for the purpose of process monitoring.

In the context of this paper model based methods are central. Model-based methods for fault-diagnosis have been reviewed by Venkatasubramanian et al. ${ }^{13,114}$ To support qualitative model based monitoring and emergency planning a modelling methodology which can support causal and consequential reasoning is needed. Several such systems have been suggested; ${ }^{113}$ one particularly popular form is the signed directed graph which is qualitative, 
however, often the knowledge representation used is structure centered ${ }^{115}$ rather than functionality centered. Another class of graphs, bond graphs constitute an interesting originally quantitative graphical representation of a physical dynamic system which cover multiple energy domains seamlessly. Bond graphs is an interesting model type in that they enable causal reasoning. ${ }^{116}$ Due to their support of causal reasoning bond graphs have also been used for monitoring purposes. However, bond graphs does not include explicit concepts for handling the purpose structure of the plant. This is in contrast to MFM which is based on function as purpose of the object, while bond graphs are based on function as effect (of the input).

In the following focus will address one qualitative model based approach for supporting plant operations based on functional concepts which are coherent with human cognition since process plants are complex socio-technical systems.

\section{Plant Monitoring}

Qualitative functional models may be used to monitor plant operations, i.e. to diagnose the origin of a process deviation and to predict consequences of process deviations based on the ability of the plant to achieve the desired production and quality purposes. A modelling methodology developed especially for these purposes has been proposed by $\operatorname{Lind}^{117}$ and have since been extended with reasoning tools ${ }^{118}$ and a tutorial by Zhang and Lind. ${ }^{119}$ The main purpose of Multilevel Flow Modelling (MFM) is to represent knowledge of objectives and functions of complex processes for the purpose of monitoring process operation in relation to the operational goal for the plant. MFM provides concepts, a methodology and a formal language for expression of design intentions and support of operations. It does this by using the basic principles of means-end analysis and identification of relations between plant objectives and the causal factors i.e. functions (the means) and the plant physical structure. The MFM language provides a formalized representation which supports reasoning about causes and consequences of disturbances and planning of emergency responses. ${ }^{17}$ 
In MFM functionality is represented by interconnected elementary flow and control functions to form functional structures representing a particular purpose oriented view of the system. The mass and energy flow functions include source, sink, transport, storage, balance, barrier, separation, conversion, and distribution. The control functions include steer, trip, regulate and suppress. The flow and control functions are related by means-end and influence relations representing purpose related dependencies between objectives and functions, these include produce, maintain, suppress, destroy, mediate and producer-product. The name of the last relation indicates that the role of the material has changed from being produced in one flow structure to having an active role, i.e. product function in the other flow structure, e.g. being a promotor of a gene in a bioprocess. The functional structures and their relations form a hyper-graph structure which comprise a comprehensive representation of the functional organization of the system. The transported materials are represented through their state of matter, i.e. solid, liquid or gas. Specific components are indicated through their role in the bulk transport phase. The main features are that MFM:

- represents systems and their interactions on several levels of abstraction

- supports cause-consequence reasoning

- provides formalized representation of operational situations and

- includes concepts which are coherent with human cognition

Therefore results of MFM reasoning can be communicated to designers and operators. The above four features indicate that MFM can deal with realistic engineering problems in different domains, such as cause identification of abnormal situations (fault diagnosis), situation awareness, operational planning, e.g. of alarm responses.

Returning to the gas/oil platform example, then an illustrative simple MFM model for the mass and energy flow structures of the three phase separation of the inflow stream from the well manifold is shown in Figure 7 where the 
generally accessible Egolf MFM editor and reasoning platform ${ }^{120}$ has been used. Note that no instrumentation is included, and thereby no control is shown, but that the objective of the model is included and can be used in reasoning about deviations. The MFM model simply illustrates that from the mixed inflow stream first the gas and liquid are separated, then the oil and water are separated and the oil is further dehydrated. All product streams flow to further processing. It is important to note the causal relations, i.e. the influencer and participant relations between the flow functions and the transports. It is these relations together with the means-end relations that form the basis for enabling reasoning in MFM. This simple qualitative functional model of the three phase separation on the topside of an oil production platform can be used for at least two purposes: to identify the phenomena ${ }^{82}$ involved in the topside processing for phenomena based optimization of the topside design, and for monitoring topside platform operating performance. The model only indicates three phenomena a gas-liquid separation, which removes the natural gas from the liquids, a liquid-liquid separation, which separate water from the crude oil and a further dehydration separation of the crude oil. With this information and knowledge of the operating envelope of the 3-phase separator an optimization problem can be stated to find the best phenomena structure for the separator. ${ }^{82}$

The MFM model represents the system by using means-end and part-whole decompositions as outlined in Figure 5, and describes not only the purposes and functions of the system but also the causal relations between elements of the system. ${ }^{20,121}$ Thus MFM is a tool for causal reasoning and for development of fault trees or consequence trees when evaluating fault scenarios or investigating accidents. In such applications MFM can provide operator decision support in abnormal operating situations as illustrated on a PWR nucelar reactor. ${ }^{122,123}$ This includes diagnosis of disturbances by finding their causes and consequences and the derivation of possible plans of emergency for disturbance mitigation. ${ }^{17}$ The development of 
emergency plans is illustrated in Figure 8. Qualitative functional thinking can also be used for planning start-up sequences, ${ }^{124}$ similarly MFM can be used for modelling operating modes. ${ }^{122}$ MFM is also applicable in the plant design phase as a tool for systematic specification of plant design objectives and functions and for transfer of design intent between process and automation design teams. MFM has also been used for Hazop studies, where it is demonstrated that MFM and its tools can provide more consistent and complete analyses compared with traditional manual methods. ${ }^{125,126} \mathrm{Wu}^{126}$ developed an MFM model of a three phase separator, which is a central operation in the gas/oil platform example. That work illustrates how an MFM model at different levels of detail can be used in HAZOP studies and for reasoning about causes and consequences in a model assisted HAZOP.

Simple examples of causal and consequence reasoning in the case of a low deviation in the oil flow function of the mass flow structure in Figure 7 are presented in Figures 9 and 10 respectively. The potential causes are low mass transport from the well or gas flow. The potential consequences are that the oil-water separation fail or the water flow is high. According to $\mathrm{Wu}$ et.al ${ }^{126}$ a possible protection for this deviation is a level controller of the interface in the water compartment. However, industrial experience indicate that level controllers are prune to failure, and hence the addition of an independent protection system may be called for by the HAZOP team.

An important issue is related to validation of qualitative functional models, ${ }^{127}$ where it is desirable to assess whether the intended system purpose is properly represented as well as whether the model represents the system behavior sufficiently well, i.e. both the means-end and the part-whole dimensions have to be validated.

A conceptually related approach to MFM for hazard identification (HAZID) is the blended HAZID ${ }^{128}$ where HAZOP and and Failure Mode and Effects analysis (FMEA) are combined. This interesting approach also recognizes that process system are complex socio-technical systems. The key aspect in this approach is that a component and a function driven HAZID 
method are blended in an attempt to obtain higher coverage of hazards at the price of requiring the users to be highly skilled and have deep understanding of the causal dependencies and the component failure mode, consequently further development of computer-aided tools was initiated.

With the increasing importance of biotechnology also for process systems engineering it is relevant to point out that functional modelling also can have a significant role in modelling the functional behaviour of microorganisms as investigated for MFM applied in modelling of a specific operon in E-coli. ${ }^{129,130}$

It has been shown generally that qualitative functional process models can be converted to signed directed graphs by translating implicit causalities to explicit ones. ${ }^{131}$ Thereby the extensively researched field of fault diagnosis using signed directed graphs is directly accessible for the functional modelling community. This provides the opportunity to integrate such research into the development of functional reasoning systems with minimal effort. On the other hand the signed directed graph based fault analysis community can profit from using functional models by exploiting the existing functional modeling concepts that are designed to identify causal structures in specific industrial applications. Following such guidelines ensures consistent model design and thereby consistent design of the signed directed graphs used for the analysis. In addition follows that the opportunity to express the diagnosis output in a functional modeling framework designed for the evaluation by process experts facilitates communication in the respective target industry. ${ }^{131}$

MFM models the objectives and functions of complex systems with different relation types in the means-end and part-whole dimensions. Therefore the developed model can be used to analyze dependency relations between different functions and objectives. Such reasoning with MFM models is based on generic cause-effect relations. ${ }^{121,132,133}$ MFM is therefore very effective for building knowledge bases for model based expert systems. The need to develop rules for reasoning about causes and effects which is a characteristic of rule based systems is eliminated entirely and the modelling effort is reduced to building the qualitative functional 
model using the defined functions and relations.

MFM is used as the underlying supporting modeling and diagnosing method for commercial purposes in collaboration with industry. The purpose is to develop an on-line operator decision support system for handling alarm floods through dynamically generating cause and consequence analysis of plant upsets and derive action plans to the operator for unplanned upsets and abnormal events, while at the same time displaying the root cause and future consequences of the situation.

\section{Discussion}

The presentation of functional modelling, i.e. modelling for the purpose of the object under investigation, leads to principles for development of qualitative and quantitative physicochemical models. It is demonstrated that modelling hermeneutics forms an essential part in these types of functional model development, as illustrated in Figures 1 - 3, where functional modelling hermeneutics incorporates:

Functional modelling hermeneutics: Translating the system goal (or subgoal) into desired system properties, identifying the needed theory to model the system to represent these properties and identifying the evaluation criteria for system purpose and performance

This concept is essentially just a formalization of what as been taking place in process systems engineering throughout the last half century when modelling for the purpose of the object under investigation. When there is an available theory to model the system under investigation and it is possible to translate the system goal into desired properties then it is possible to apply reverse engineering and thereby obtain design proposals which are among the best possible. Such designs can then be tested experimentally and thereby validated. Such tests are also part of the functional modelling hermeneutics. In this paper the term hermeneutics is selected to indicate that the first important step is the translation of the 
system goal (or sub-goal) into properties in such a way that these may be modelled in so far as possible in order to exploit reverse engineering methodology.

The application and analysis of functional modelling in product and process design reveals that hermeneutics also forms an essential part thereof as indicated by the concept of:

Functional design hermeneutics: Translating an overall product/process design goal into a set of sub-goals where each sub-goal can form the basis for a task in the design procedure. Identifying the needed theoretical bases, if available, to represent the sub-goal property/-ies and identifying the evaluation criteria for system purpose and performance.

Thereby the workflow of a design activity involves development of a subgoal structure where each subgoal can define a task. In some cases this task may be subject to modelling where the modelling hermeneutics can be applied. Although the tools for product design are able to facilitate the design of single-species small molecules and blends, for other product types, mainly correlations and/or collected data are commonly employed. Instead predictive models are needed. However, the issue of modelling properties and product function is not simple as pointed out by several authors. ${ }^{42,65,67}$ The difficulty is that different property sets define the properties and functions of different products. Furthermore for structured products the needed property models do not exist in a form that lends itself to computer-aided design. ${ }^{45}$ This is also true for many large molecular products, e.g. active pharmaceutical ingredients, membranes, and catalysts, where the molecular structure-property relationship plays an important role. A limiting aspect is lacking knowledge and understanding of many phenomena at the molecular and submolecular level. Therefore the science of product function-microstructure relationships can provide significant future contributions. For functional catalyst design also directed evolution can be expected to play a significant role in development of efficient enzymes for many different reactions through dilligent application of evolution for reaching very efficient catalysts. ${ }^{134,135}$

The above discussion is also reflected by Wintermantel, ${ }^{136}$ who states that, the 'scientific 
part' of chemical engineering consists exactly in breaking down complex formulated systems (products) into subsystems, which are then described using our understanding of fundamental chemical and physical processes. The 'engineering part' of chemical engineering consists in using the gained knowledge (even if incomplete) in the design of a product which matches the desired characteristics, and of a process which is capable of producing the desired product. Methods and tools that make it possible to fully integrate individual steps to design a final product for its application purpose should be developed, in order to reach a final design and/or evaluation of the overall system. Consequently the proposed hermeneutic concepts for functional modelling and design constitute essential concepts within chemical engineering science.

The applications of functional modelling suggests that qualitative modelling for the purpose of the object under investigation forms the basis for many different aspects of process modelling supported by the ability to reason about causes and consequences of disturbances. This is illustrated in Figure 8 where, in addition to the fault trees and consequence trees developed through causal reasoning, reasoning about an operational goal enables development of goal trees as part of emergency plan development. Functional modelling also is used for suggesting phenomena building blocks to enable process integration, however this coupling is not explicitly shown in Figure 8. This paper suggests that functional modelling hermeneutics also forms the basis for quantitative physicochemical modelling for the purpose of the object under investigation. However, the quantitative physicochemical models only can support reasoning indirectly through repeated solution. Several applications of quantitative modelling are shown in Figure 8. These applications include process intensification based on phenomena building blocks (PPB) and the derived simultaneous phenomena building blocks (SPB) the quantitative representation of which is based on (reverse) simulation of static quantitative models. Further applications include design validation based on rigorous process simulation and model based control (MPC) design validation based on dynamic process models. The finding that functional modelling hermeneutics for the purpose of the 
object under investigation forms the basis for many different aspects of process modelling comes as no surprise since the different functional process modelling applications have a common root, they are all based upon first principles, i.e. the conservation laws plus some relatively simple thermodynamics; while as illustrated in Figure 6 the quantitative property models with more rigorous thermodynamics are essential for quantitative product/process model development. Consequently it may prove beneficial to base new modelling tools on functional modelling hermeneutics as common base for the purpose of development of tools for product/process systems engineering which are sufficiently flexible to cover the large and diverse set of tasks. Clearly such a decision needs further development of the qualitative tools. But as shown by Linds group ${ }^{133}$ then basing the development upon a formal language enables exploitation of qualitative reasoning within several important areas, such as alarm handling, fault detection, consequence reasoning and planing of emergency response actions. With the impressive range of methods and tools that have been developed through the last sixty-seventy years in process systems engineering many important product/process problems have been and are being solved. However, the challenges related to the exploitation of the available, but limited resources on the planet poses significant opportunities for advances in fundamental science and technology. ${ }^{5,81}$ With further developments in predictive property modelling capabilities, e.g. ${ }^{100}$ it becomes possible to design novel molecules and chemical products for specific intended purposes with limited need for experimental data except within catalyst design where directed evolution is expected to enable application of functional thinking. These developments suggest that it will be possible to further the applications of functional modelling for the intended purpose of the object under investigation and thereby improve the ability to obtain designs which are among the best available wrt. sustainability, safety and other performance criteria.

The application of functional modelling for monitoring and safety related issues constitute an important development for facilitating tools for qualitative reasoning which are coherent with human cognition. Thereby monitoring tools such as alarm systems and mitigation 
planning in the event of a fault better can suit the needs of the operations, both in avoiding negative situations, such as abnormal shutdown, releases etc, and in identifying opportunities for improvement of operations. Furthermore, the formalized functional modelling language developed by Lind ${ }^{117-119}$ MFM is very effective for building knowledge bases for model based expert systems. The need to develop rules for reasoning about causes and effects which is a characteristic of rule based systems is eliminated entirely and the modelling effort is reduced to building a qualitative functional model for the intended purpose of the object under investigation using the defined functions and relations.

\section{Conclusions}

The paradigm of functional modelling for the intended purpose of the object under investigation is developed for product and process systems engineering. This paradigm is based upon an underlying theoretical basis. For product modelling the underlying theory is thermodynamics modelled using preferably approximate group contribution methods or mechanistic, i.e. quantum mechanics methods in order to obtain predictive methods. If these models are not available correlation approaches may be used, but then the predictive power is limited. For process modelling the basis is first principles engineering, i.e. conservation laws combined with modeling of the involved molecules. The general modelling purposes are product/process design, which in this paper is represented by molecular design, process design, process intensification and process monitoring. The paper illustrates different application aspects of functional modelling within these areas. The applications within process modelling illustrates that a functional process model hermeneutics may be viewed as core basis, i.e. a common building block as illustrated in Figure 8 for both qualitative functional and quantitative computational physicochemical process models. This observation may prove useful in

development of future flexible tools for product and process systems engineering. ${ }^{5,137,138}$ The applications within process monitoring, where a formalized qualitative functional modelling 
language, MFM is in focus, illustrates the potential power of such formalization through the possibility of using computer aided causal reasoning tools for mode changes such as start-up planning, HAZOP analysis assistance, fault detection and consequence reasoning as well as planning of mitigating actions. Potentially such tools can be most useful also for online support of operators.

The functional modelling paradigm analysis leads to proposing the concepts of hermeneutics of functional modelling and of product/process design. These concepts are suggested as essential for chemical engineering science and thereby also for process systems engineering in its further development of the sustainable and safe solutions which our planet needs.

\section{Acknowledgement}

One of the authors (Niels) thanks Sirish Shah and the late D.Grant Fisher for providing the opportunity many years ago to dive into process control in a very inspiring and international group at University of Alberta leading to a challenging career in industry and academia. The two first authors gratefully received partial funding from the Danish Hydrocarbon Research and Technology Centre.

\section{References}

(1) Hangos, K.; Cameron, I. Process modelling and model analysis; Process Systems Engineering; Elsevier, 2001; Vol. 4.

(2) Klatt, K.-U.; Marquardt, W. Perspectives for process systems engineering-Personal views from academia and industry. Comput. Chem. Eng. 2009, 33, 536-550.

(3) Chen, Q.; Grossmann, I. E. Recent developments and challenges in optimization-based process synthesis. Annu. Rev. Chem. Biomol. Eng. 2017, 8, 249-283. 
(4) Gernaey, K. V.; Gani, R. A model-based systems approach to pharmaceutical productprocess design and analysis. Chem. Eng. Sci. 2010, 65, 5757-5769.

(5) Tula, A. K.; Eden, M. R.; Gani, R. Time for a new class of methods and computer aided tools to address the challenges facing us? Chemical Engineering Transactions. 2018; pp 7-12.

(6) Erden, M. S.; Komoto, H.; van Beek, T. J.; D’Amelio, V.; Echavarria, E.; Tomiyama, T. A review of function modeling: Approaches and applications. Artif. Intell. Eng. Des. Anal. Manuf. 2008, 22, 147-169.

(7) Vermaas, P. E. The coexistence of engineering meanings of function: Four responses and their methodological implications. Artif. Intell. Eng. Des. Anal. Manuf. 2013, 27, $191-202$.

(8) Bertalanffy, L. V. O. N. An outline of general system theory. Br. J. Philos. Sci. 1950, I, $134-165$.

(9) Schon, D. A. The reflective prationer: How professionals think in action; Basic Books, 1983.

(10) Simon, H. A. The Sciences of the artificial; MIT Press: Cambridge, MA, 1969.

(11) Kearney, R. Paul Ricoeur and the hermeneutics of translation. Research in Phenomenology 2007, 37, 147-159.

(12) hermeneutic | Definition of hermeneutic in English by Oxford Dictionaries. https: //en.oxforddictionaries.com/definition/hermeneutic, Accessed: 2019-3-13.

(13) Wimsatt, W. C. Teleology and the logical structure of function statements. Studies in History of Philosophy of Science 1972, 3, 1-80.

(14) teleology | Definition of teleology in English by Oxford Dictionaries. https://en . oxforddictionaries.com/definition/teleology, Accessed: 2019-3-13. 
(15) Umeda, Y.; Tomiyama, T. FBS Modeling : modeling scheme of function for conceptual design. Proc. Working Papers of the 9th Int. Workshop on Qualitative Reasoning About Physical Systems. 2003; pp 271-278.

(16) De Kleer, J.; Brown, J. S. A qualitative physics based on confluences. Artif. Intell. 1984, 24, 7-83.

(17) Gofuku, A.; Tanaka, Y. Application of a derivation technique of possible counter actions to an oil refinery plant. 1999.

(18) Petersen, J. Causal reasoning based on MFM. Proceedings of Cognitive Systems Engineering in Process Control. 2000.

(19) Lind, M.; Zhang, X. Functional modelling for fault diagnosis and its appliction for NPP. Nuclear Engineering and Technology 2014, 46, 753-772.

(20) Zhang, X.; Lind, M.; Ravn, O. Consequence reasoning in multilevel flow modelling. IFAC Proceedings Volumes 2013, 46, 187-194.

(21) Lind, M. An introduction to multilevel flow modeling. International Electronic Journal of Nuclear Safety and Simulation 2011, 2, 22-32.

(22) Stephanopoulos, G.; Henning, G.; Leone, H. MODEL.LA. A modeling language for process engineering-I. The formal framework. Comput. Chem. Eng. 1990, 14, 813846.

(23) Andersson, M. An object-oriented language for model representation. Ph.D. thesis, Lund Institute of Technology, 1990.

(24) Piela, P. C.; Epperly, T. G.; Westerberg, K. M.; Westerberg, A. W. ASCEND: an object-oriented computer environment for modeling and analysis: The modeling language. Comput. Chem. Eng. 1991, 15, 53-72. 
(25) Priesig, H. A. An object-oriented computer-aided modelling tool. Foundations of computer-aided process design. 1995; pp 328-331.

(26) Vazquez-Roman, R. Computer aids for process model building. Ph.D. thesis, University of London, 1992.

(27) Preisig, H. A.; Elve, A. T. Ontology construction for multi-network models. Computer Aided Chemical Engineering 2016, 39, 1087-1092.

(28) Gani, R.; Hytoft, G.; Jaksland, C.; Jensen, A. K. An integrated computer aided system for integrated design of chemical processes. Comput. Chem. Eng. 1997, 21, 1135-1146.

(29) Erikøi, L. Comparison of Aspen HYSYS and Aspen Plus simulation of CO2 absorption into MEA from atmospheric gas. Energy Procedia 2012, 23, 360-369.

(30) Winkel, M. L.; Zullo, L. C.; Verheijen, P. J. T.; Pantelides, C. C. Modelling and simulation of the operation of an industrial batch plant using gPROMS. Comput. Chem. Eng. 1995, 19, 571-576.

(31) Jaksland, C. A.; Gani, R.; Lien, K. M. Separation process design and synthesis based on thermodynamic insights. Chem. Eng. Sci. 1995, 50, 511-530.

(32) Constantinou, L.; Gani, R. New group contribution method for estimating properties of pure compounds. AIChE J. 1994, 40, 1697-1710.

(33) Austin, N. D.; Sahinidis, N. V.; Trahan, D. W. Computer-aided molecular design: An introduction and review of tools, applications, and solution techniques. Chem. Eng. Res. Des. 2016, 116, 2-26.

(34) Morejon, J. M.; Gani, R. Group contribution based estimation of pure component properties. Fluid Phase Equilib. 2001, 183, 183-208. 
(35) Gani, R.; Harper, M., Peter M.and Hostrup Automatic Creation of Missing Groups through Connectivity Index for Pure-Component Property Prediction. Ind. Eng. Chem. Res. 2005, 44, 7262-7269.

(36) Hukkerikar, A. S.; Kalakul, S.; Sarup, B.; Young, D. M.; Sin, G.; Gani, R. Estimation of environment-related properties of chemicals for design of sustainable processes: development of group-contribution $+(\mathrm{GC}+)$ property models and uncertainty analysis. J. Chem. Inf. Model. 2012, 52, 2823-2839.

(37) Jhamb, S.; Liang, X.; Gani, R.; Hukkerikar, A. S. Estimation of physical properties of amino acids by group-contribution method. Chem. Eng. Sci. 2018, 175, 148-161.

(38) Cussler, E. L.; Moggridge, G. D. Chemical product design; Cambridge University Press, 2011.

(39) Samudra, A. P.; Sahinidis, N. V. Optimization-based framework for computer-aided molecular design. AIChE J. 2013, 59, 3686-3701.

(40) Bagajewicz, M. J. On the role of microeconomics, planning, and finances in product design. AIChE J. 2007, 53, 3155-3170.

(41) Gani, R. Chemical product design: challenges and opportunities. Comput. Chem. Eng. 2004, 28, 2441-2457.

(42) Ng, L. Y.; Chong, F. K.; Chemmangattuvalappil, N. G. Challenges and opportunities in computer-aided molecular design. Comput. Chem. Eng. 2015, 81, 115-129.

(43) Gani, R.; Brignole, E. A. Molecular design of solvents for liquid extraction based on UNIFAC. Fluid Phase Equilib. 1983, 13, 331-340.

(44) Joback, K. G.; Stephanopoulos, G. Designing molecule possessing desired physical property values. Proceedings of Foundation of Computer-Aided Process Design. Amsterdam, 1989; p 363. 
(45) Hill, M. Product and process design for structured products. AIChE J. 2004, 50, $1656-1661$.

(46) Wibowo, C.; Ng, K. M. Product-oriented process synthesis and development: Creams and pastes. AIChE J. 2001, 47, 2746-2767.

(47) Harper, P. M.; Gani, R. Computer aided tools for design/selection of environmentally Friendly substances. In Process Design Tools for Environment, chap.15; 2000; pp 371-404.

(48) Harper, P. M.; Hostrup, M.; Gani, R. A hybrid CAMP method. In Computer Aided Molecular Design: Theory and Practice; Achenie, L. E. K., Gani, R., Venkatasubramanian, V., Eds.; Elsevier Science B.V., 2003; pp 129-165.

(49) Gani, R.; Jimenez-Gonzalez, C.; Constable, D.; Shafique Bashir, M. Solvents in organic synthesis: Replacement and multi-step reaction systems. 2008.

(50) Satyanarayana, K. C.; Abildskov, J.; Gani, R.; Tsolou, G.; Mavrantzas, V. G. Multiscale modelling for computer aided polymer design. Proceedings of 10th International Symposium on Process Systems Engineering - PSE2009. 2009; pp 213-218.

(51) Solvason, C. C.; Chemmangattuvalappil, N. G.; Eden, M. R. Decomposition techniques for multiscale structured product design: Molecular synthesis. In Computer Aided Chemical Engineering; Jeżowski, J., Thullie, J., Eds.; Elsevier, 2009; Vol. 26; pp 153158.

(52) Shelley, M. D.; El-Halwagi, M. M. Component-less design of recovery and allocation systems: a functionality-based clustering approach. Comput. Chem. Eng. 2000, 24, $2081-2091$.

(53) Odele, O.; Macchietto, S. Computer aided molecular design: a novel method for optimal solvent selection. Fluid Phase Equilib. 1993, 82, 47-54. 
(54) Venkatasubramanian, V.; Chan, K.; Caruthers, J. M. Computer-aided molecular design using genetic algorithms. Comput. Chem. Eng. 1994, 18, 833-844.

(55) Vaidyanathan, R.; El-Halwagi, M. Computer-aided design of high performance polymers. J. Elastomers Plast. 1994, 26, 277-293.

(56) Duvedi, A. P.; Achenie, L. E. K. Designing environmentally safe refrigerants using mathematical programming. Chem. Eng. Sci. 1996, 51, 3727-3739.

(57) Maranas, C. D. Optimal molecular design under property prediction uncertainty. AIChE J. 1997, 43, 1250-1264.

(58) Raman, V. S.; Maranas, C. D. Optimization in product design with properties correlated with topological indices. Comput. Chem. Eng. 1998, 22, 747-763.

(59) Camarda, K. V.; Maranas, C. D. Optimization in polymer design using connectivity indices. Ind. Eng. Chem. Res. 1999, 38, 1884-1892.

(60) Sahinidis, N. V.; Tawarmalani, M.; Yu, M. Design of alternative refrigerants via global optimization. AIChE J. 2003, 49, 1761-1775.

(61) Sinha, M.; Achenie, L. E. K.; Gani, R. Blanket wash solvent blend design using interval analysis. Ind. Eng. Chem. Res. 2003, 42, 516-527.

(62) Karunanithi, A. T.; Achenie, L. E. K.; Gani, R. A new decomposition-based computeraided molecular/mixture design methodology for the design of optimal solvents and solvent mixtures. Ind. Eng. Chem. Res. 2005, 44, 4785-4797.

(63) Karunanithi, A. T.; Achenie, L. E. K.; Gani, R. A computer-aided molecular design framework for crystallization solvent design. Chem. Eng. Sci. 2006, 61, 1247-1260.

(64) Kalakul, S.; Zhang, L.; Fang, Z.; Choudhury, H. A.; others, Computer aided chemical product design-ProCAPD and Tailor-made blended products. Comput. Chem. Eng. 2018, 116, 37-55. 
(65) Gani, R.; Abildskov, J.; Kontogeorgis, G. M. Chapter 14: Application of property models in chemical product design. In Computer Aided Chemical Engineering; Kontogeorgis, G. M., Gani, R., Eds.; Elsevier, 2004; Vol. 19; pp 339-369.

(66) Conte, E.; Sansonetti, S.; Crafts, P. A.; Gani, R. Rational design of pharmaceutical and other Liquid formulations. 2011 AIChE Annual Meeting. 2011.

(67) Zhang, L.; Babi, D. K.; Gani, R. New vistas in chemical product and process design. Annu. Rev. Chem. Biomol. Eng. 2016, 7, 557-582.

(68) Siirola, J. J. Industrial applications of chemical process synthesis. In Advances in Chemical Engineering; Anderson, J. L., Ed.; Academic Press, 1996; Vol. 23; pp 1-62.

(69) Siirola, J. J.; Powers, G. J.; Rudd, D. F. Synthesis of system designs: III. Toward a process concept generator. AIChE J. 1971, 17, 677-682.

(70) Douglas, J. M. A hierarchical decision procedure for process synthesis. AIChE J. 1985, 31, 353-362.

(71) Cabezas, H.; Argoti, A.; Friedler, F.; Mizsey, P.; Pimentel, J. Design and engineering of sustainable process systems and supply chains by the P-graph framework. Environ. Prog. Sustain. Energy 2018, 37, 624-636.

(72) Raman, R.; Grossmann, I. E. Relation between MILP modelling and logical inference for chemical process synthesis. Comput. Chem. Eng. 1991, 15, 73-84.

(73) Friedler, F.; Tarján, K.; Huang, Y. W.; Fan, L. T. Graph-theoretic approach to process synthesis: axioms and theorems. Chem. Eng. Sci. 1992, 47, 1973-1988.

(74) Ierapetritou, M. G.; Acevedo, J.; Pistikopoulos, E. N. An optimization approach for process engineering problems under uncertainty. Comput. Chem. Eng. 1996, 20, 703709. 
(75) Hostrup, M.; Harper, P. M.; Gani, R. Design of environmentally benign processes: integration of solvent design and separation process synthesis. Comput. Chem. Eng. 1999, 23, 1395-1414.

(76) Bek-Pedersen, E.; Hostrup, M.; Gani, R. Using driving force based separation efficiency curves within an Integrated system for process synthesis/design. In Computer Aided Chemical Engineering; Elsevier, 2000; Vol. 8; pp 955-âĂŞ960.

(77) Bek-Pedersen, E.; Gani, R. Design and synthesis of distillation systems using a drivingforce-based approach. Chemical Engineering and Processing: Process Intensification 2004, 43, 251-262.

(78) d'Anterroches, L.; Gani, R. Group contribution based process flowsheet synthesis, design and modelling. Fluid Phase Equilib. 2005, 228-229, 141-146.

(79) Tula, A. K.; Eden, M. R.; Gani, R. Process synthesis, design and analysis using a process-group contribution method. Comput. Chem. Eng. 2015, 81, 245-259.

(80) Van Gerven, T.; Stankiewicz, A. Structure, energy, synergy, time - the fundamentals of process intensification. Ind. Eng. Chem. Res. 2009, 48, 2465-2474.

(81) Sitter, S.; Chen, Q.; Grossmann, I. E. An overview of process intensification methods. Curr. Opin. Chem. Eng. 2019,

(82) Lutze, P.; Babi, D. K.; Woodley, J. M.; Gani, R. Phenomena based methodology for process synthesis incorporating process intensification. Ind. Eng. Chem. Res. 2013, 52, 7127-7144.

(83) Papalexandri, K. P.; Pistikopoulos, E. N. Generalized modular representation framework for process synthesis. AIChE J. 1996, 42, 1010-1032.

(84) Freund, H.; Sundmacher, K. Towards a methodology for the systematic analysis and design of efficient chemical processes: Part 1. From unit operations to elementary 
process functions. Chemical Engineering and Processing: Process Intensification 2008, 47, 2051-2060.

(85) Siirola, J. J. Strategic process synthesis: Advances in the hierarchical approach. Comput. Chem. Eng. 1996, 20, S1637-S1643.

(86) Peschel, A.; Freund, H.; Sundmacher, K. Methodology for the design of optimal chemical reactors based on the concept of elementary process functions. Ind. Eng. Chem. Res. 2010, 49, 10535-10548.

(87) Rong, B.-G.; Kolehmainen, E.; Turunen, I. Methodology of conceptual process synthesis for process intensification. In Computer Aided Chemical Engineering; Braunschweig, B., Joulia, X., Eds.; Elsevier, 2008; Vol. 25; pp 283-288.

(88) Arizmendi-Sánchez, J. A.; Sharratt, P. N. Phenomena-based modularisation of chemical process models to approach intensive options. Chem. Eng. J. 2008, 135, 83-94.

(89) Lutze, P.; Babi, D. K.; Woodley, J. M.; Gani, R. Phenomena based methodology for process synthesis incorporating process intensification. Ind. Eng. Chem. Res. 2013, 52, 7127-7144.

(90) Carvalho, A.; Matos, H. A.; Gani, R. SustainPro-A tool for systematic process analysis, generation and evaluation of sustainable design alternatives. Comput. Chem. Eng. 2013, 50, 8-27.

(91) Kalakul, S.; Malakul, P.; Siemanond, K.; Gani, R. Integration of life cycle assessment software with tools for economic and sustainability analyses and process simulation for sustainable process design. J. Clean. Prod. 2014, 71, 98-109.

(92) Babi, D. K.; Holtbruegge, J.; Lutze, P.; Gorak, A.; Woodley, J. M.; Gani, R. Sustainable process synthesis-intensification. Comput. Chem. Eng. 2015, 81, 218-244. 
(93) Tula, A. K.; Babi, D. K.; Bottlaender, J.; Eden, M. R.; Gani, R. A computer-aided software-tool for sustainable process synthesis-intensification. Comput. Chem. Eng. 2017, 105, 74-95.

(94) Rivas, D. F.; Castro-Hernández, E.; Villanueva Perales, A. L.; van der Meer, W. Evaluation method for process intensification alternatives. Chemical Engineering and Processing - Process Intensification 2018, 123, 221-232.

(95) Kuhlmann, H.; Skiborowski, M. Optimization-based approach To process synthesis for process intensification: General approach and application to ethanol dehydration. Ind. Eng. Chem. Res. 2017, 56, 13461-13481.

(96) Demirel, S. E.; Li, J.; Hasan, M. M. F. Systematic process intensification using building blocks. Comput. Chem. Eng. 2017, 105, 2-38.

(97) Demirel, S. E.; Li, J.; Hasan, M. M. F. A general framework for process synthesis, integration, and intensification. Ind. Eng. Chem. Res. 2019,

(98) Eden, M. R.; Jørgensen, S. B.; Gani, R.; El-Halwagi, M. M. A novel framework for simultaneous separation process and product design. Chemical Engineering and Processing: Process Intensification 2004, 43, 595-608.

(99) Conte, E.; Gani, R.; Ng, K. M. Design of formulated products: A systematic methodology. AIChE J. 2011, 57, 2431-2449.

(100) Scheffczyk, J.; Schäfer, P.; Fleitmann, L.; Thien, J.; Redepenning, C.; Leonhard, K.; Marquardt, W.; Bardow, A. COSMO-CAMPD: a framework for integrated design of molecules and processes based on COSMO-RS. Mol. Syst. Des. Eng. 2018, 3, 645-657.

(101) Rosenbrock, H. H. Design of multivariable control systems using the inverse Nyquist array. Proceedings of the Institution of Electrical Engineers 1969, 116, 1929-1936. 
(102) García, C. E.; Prett, D. M.; Morari, M. Model predictive control: Theory and practice-A survey. Automatica 1989, 25, 335-348.

(103) Qin, S. J.; Badgwell, T. A. A survey of industrial model predictive control technology. Control Eng. Pract. 2003, 11, 733-764.

(104) Mayne, D. Q. Model predictive control: Recent developments and future promise. Automatica 2014, 50, 2967-2986.

(105) Jørgensen, S. B.; Lee, J. H. Recent advances and challenges in process identification. AICHE Symp. Ser. 2001, 98, 55-74.

(106) Lin, B.; Recke, B.; Renaudat, P.; Knudsen, J.; Jørgensen, S. B. A systematic approach for soft sensor development. Comput. Chem. Eng. 2007, 31, 419-425.

(107) Sin, G.; Gernaey, K. V.; Neumann, M. B.; van Loosdrecht, M. C. M.; Gujer, W. Uncertainty analysis in WWTP model applications: a critical discussion using an example from design. Water Res. 2009, 43, 2894-2906.

(108) Kolås, S.; Foss, B. A.; Schei, T. S. Noise modeling concepts in nonlinear state estimation. J. Process Control 2009, 19, 1111-1125.

(109) Barrero, O.; Petersen, J.; Jorgensen, S. B. Multirate data assimilation in a cultivation process. IFAC Proceedings Volumes 2008, 41, 11433-11438.

(110) Mayne, D. Q.; Rawlings, J. B.; Rao, C. V.; Scokaert, P. O. M. Constrained model predictive control: Stability and optimality. Automatica 2000, 36, 789-814.

(111) Amrit, R.; Rawlings, J. B.; Biegler, L. T. Optimizing process economics online using model predictive control. Comput. Chem. Eng. 2013, 58, 334-343.

(112) Wang, J.; Yang, F.; Chen, T.; Shah, S. L. An overview of industrial alarm systems: Main causes for alarm overloading, research status, and open problems. IEEE Trans. Autom. Sci. Eng. 2016, 13, 1045-1061. 
(113) Venkatasubramanian, V.; Rengaswamy, R.; Kavuri, S. N.; Yin, K. A review of process fault detection and diagnosis: Part III: Process history based methods. Comput. Chem. Eng. 2003, 27, 327-346.

(114) Venkatasubramanian, V.; Rengaswamy, R.; Yin, K.; Kavuri, S. N. A review of process fault detection and diagnosis: Part I: Quantitative model-based methods. Comput. Chem. Eng. 2003, 27, 293-311.

(115) McCoy, S. A.; Zhou, D.; Chung, P. W. H. State-based modelling in hazard identification. Applied Intelligence 2006, 24, 263-279.

(116) Paynter, H. M.; Briggs, P. Analysis and design of engineering systems - class notes for MIT course 2.751; M.I.T. Press: Cambridge, Mass., 1961.

(117) Lind, M. Modeling goals and functions of complex industrial plants. Appl. Artif. Intell. $\mathbf{1 9 9 4 , ~ 8 , ~ 2 5 9 - 2 8 3 . ~}$

(118) Lind, M. Control functions in MFM: basic principles. Nuclear safety and simulation 2011, 2, 132-140.

(119) Zhang, X.; Lind, M. Multilevel flow modelling: A tutorial; 2017.

(120) Eldor Technology, EGolf - Eldor Goals and Functions. http://egolf . azurewebsites.net, 2019; Accessed: 2019-5-24.

(121) Petersen, J. Situation assessment of complex dynamic systems using MFM. IFAC Proceedings Volumes 2001, 34, 567-572.

(122) Lind, M.; Yoshikawa, H.; Jørgensen, S. B.; Yang, M.; Tamayama, K.; Okusa, K. Multilevel flow modeling of Monju nuclear power plant. Nuclear Safety and Simulation 2011, 2, 274-284.

(123) Lind, M.; Zhang, X. Functional modelling for fault diagnosis and its appliation for NPP. Nuclear Engineering and Technology 2014, 46, 753-772. 
(124) Eden, M. R.; Koggersbøl, A.; Hallager, L.; Jørgensen, S. B. Dynamics and control during startup of heat integrated distillation column. Comput. Chem. Eng. 2000, 24, 1091-1097.

(125) Rossing, N. L.; Lind, M.; Jensen, N.; Jørgensen, S. B. A functional HAZOP methodology. Comput. Chem. Eng. 2010, 34, 244-253.

(126) Wu, J.; Zhang, L.; Hu, J.; Lind, M.; Zhang, X.; Jørgensen, S. B.; Sin, G.; Jensen, N. An integrated qualitative and quantitative modeling framework for computer-assisted HAZOP studies. AIChE J. 2014, 60, 4150-4173.

(127) Wu, J.; Lind, M.; Zhang, X.; Jørgensen, S. B.; Sin, G. Validation of a functional model for integration of safety into process system design. Proceedings of the 25th European Symposium on Computer Aided Process Engineering. 2015; pp 293-298.

(128) Seligmann, B. J.; Németh, E.; Hangos, K. M.; Cameron, I. T. A blended hazard identification methodology to support process diagnosis. J. Loss Prev. Process Indust. 2012, 25, 746-759.

(129) Gernaey, K. V.; Lind, M.; Jørgensen, S. B. MODELLING FOR CONTROL: UNDERSTANDING ROLE AND FUNCTION OF REGULATORY NETWORKS IN MICROORGANISMS. IFAC Proceedings Volumes 2005, 38, 13-18.

(130) Gernaey, K. V.; Lind, M.; Jrgensen, S. B. Towards Understanding the Role and Function of Regulatory Networks in Microorganisms. In Computer Aided Process and Product Engineering; Puigjaner, L., Heyen, G., Eds.; Wiley-VCH Verlag GmbH: Weinheim, Germany, 2006; pp 223-264.

(131) Reinartz, C.; Kirchhüble, D.; Ravn, O.; Lind, M. Generation of signed directed graphs using functional models. 2019. 
(132) Zhang, X.; Lind, M.; Ravn, O. Consequence Reasoning in multilevel flow modelling. Proceedings of the 12th IFAC/IFIP/IFORS/IEA Symposium on Analysis, Design, and Evaluation of Human - Machine Systems. 2013.

(133) Zhang, X.; Lind, M.; Jørgensen, S. B.; Wu, J.; Karnati, P. Extending MFM function ontology for representing separation and conversion in process plants. Proceedings of the 7th International Conference on Water and Food Management. 2018.

(134) Arnold, F. H. Design by Directed Evolution. Acc. Chem. Res. 1998, 31, 125-131.

(135) Loskot, S. A.; Romney, D. K.; Arnold, F. H.; Stoltz, B. M. Enantioselective Total Synthesis of Nigelladine A via Late-Stage C-H Oxidation Enabled by an Engineered P450 Enzyme. J. Am. Chem. Soc. 2017, 139, 10196-10199.

(136) Wintermantel, K. Process and product engineering - achievements, present and future challenges. Chem. Eng. Sci. 1999, 54, 1601-1620.

(137) Lee, A.; Ghouse, J. H.; Chen, Q.; Eslick, J. C.; Siirola, J. D.; Grossman, I. E.; Miller, D. C. A flexible framework and model library for process simulation, optimization and control. In Computer Aided Chemical Engineering; Eden, M. R., Ierapetritou, M. G., Towler, G. P., Eds.; Elsevier, 2018; Vol. 44; pp 937-942.

(138) Miller, D. C.; Siirola, J. D.; Agarwal, D.; Burgard, A. P.; Lee, A.; Eslick, J. C.; Nicholson, B.; Laird, C.; Biegler, L. T.; Bhattacharyya, D.; Sahinidis, N. V.; Grossmann, I. E.; Gounaris, C. E.; Gunter, D. Next generation multi-scale process systems engineering framework. In Computer Aided Chemical Engineering; Eden, M. R., Ierapetritou, M. G., Towler, G. P., Eds.; Elsevier, 2018; Vol. 44; pp 2209-2214. 


\section{Graphical TOC Entry}

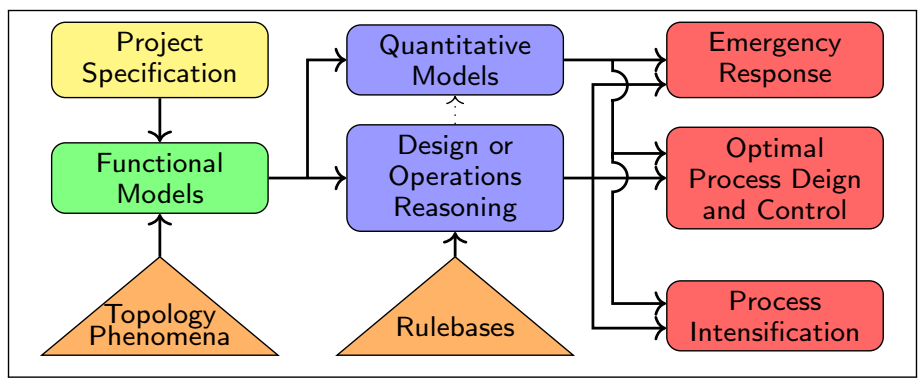




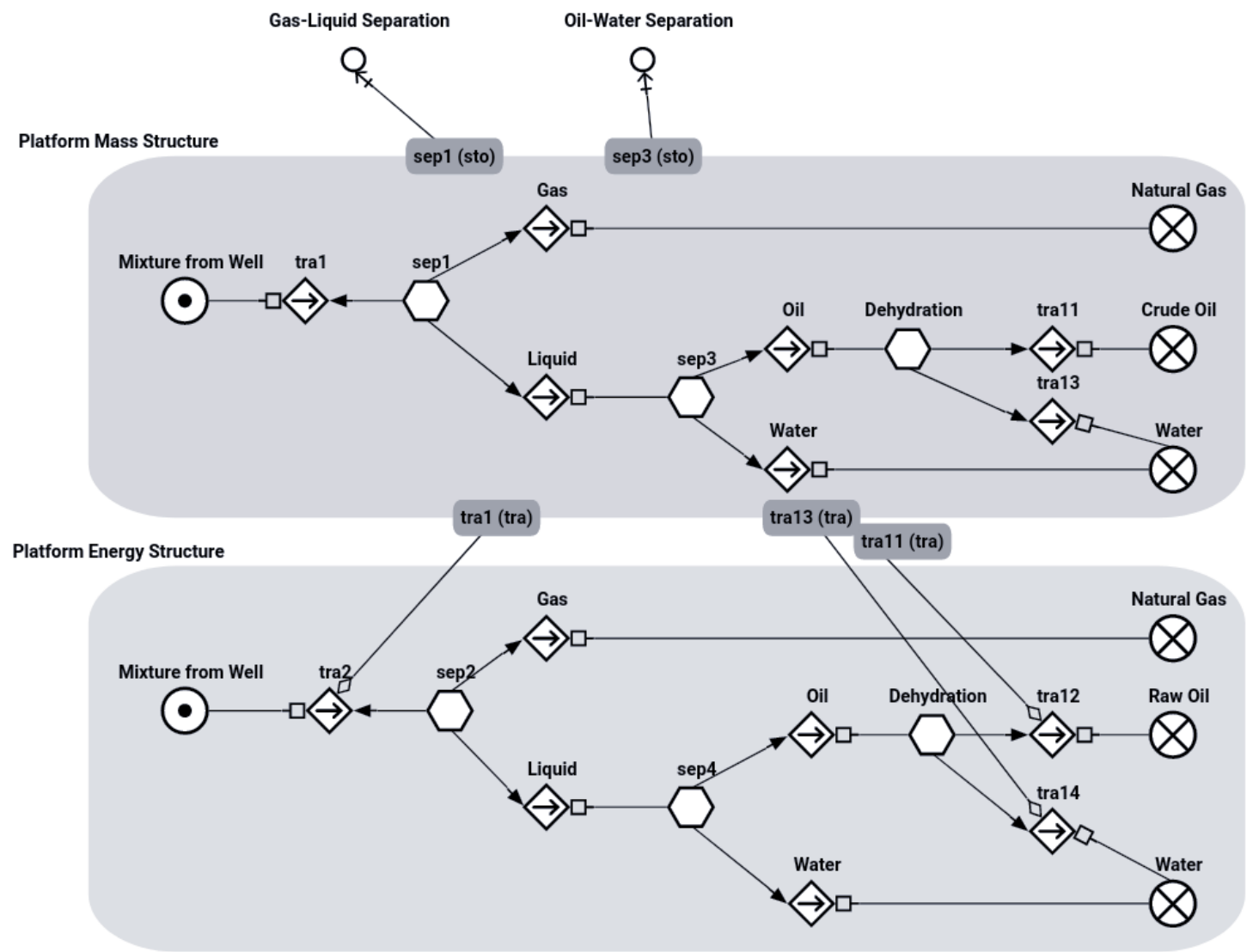

Figure 7: Mass and energy flow structures representing a simple MFM model of the topside processing on a production oil platform with the purpose of separating the stream from the wells into a natural gas stream, a raw oil stream and a water stream, as explicitly stated in the model. Generally in this structure the energy flows are mediated by the mass flows, e.g. mediate relation from the mass flow function tra1 to the energy flow function tra2. Symbol legend: (circle around a dot) source, (circle around a cross sign) sink, (diamond around an arrow) transport, and (hexagon) storage, which, in this illustrative case, is used to symbolize simple two-phase separation functionality. The causal relationships within the flow structures are defined by the connecting arrows between the flow functions: an arrow indicates an influencer relationship if the transport has the role of influencing the amount of substance transported; a diamond symbol indicates a participant relationship if the system realizing the flow has the role of passively providing or receiving substance for the transport with an arrow or a diamond. 


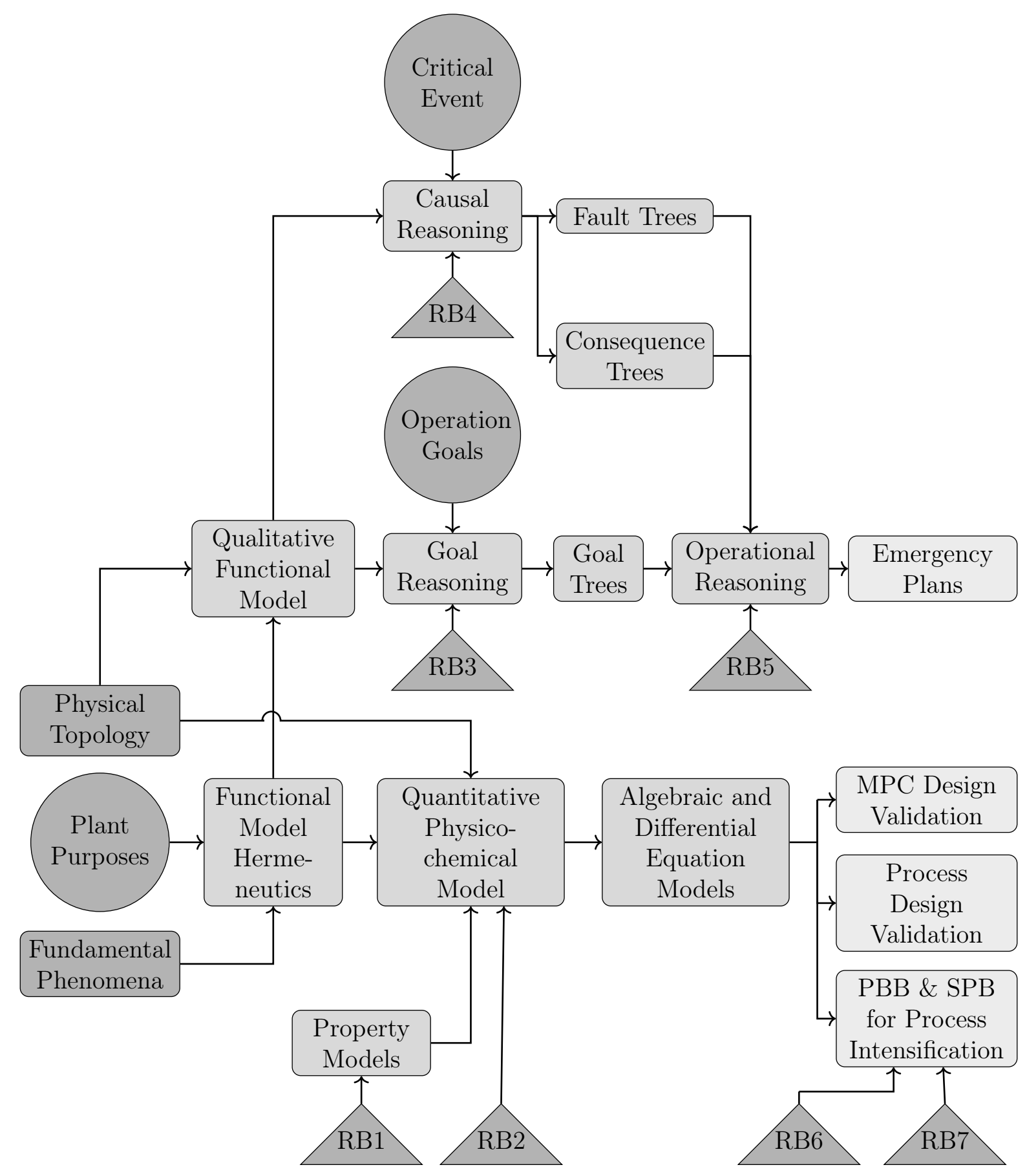

Figure 8: Functional model hermeneutics forms a common basis for qualitative functional modelling with reasoning capabilities, e.g. goal tree and emergency planing; as well as for quantitative physicochemical model synthesis for process simulation and design purposes. PBB is Phenomena and SPB Simultaneous Phenomena Building Blocks for Process Intensification, i.e. innovative process design. Symbols: Dark gray indicate design input in the form of phenomena, topology, purpose, operation goals and critical events plus a number of rule bases (RB). Very light gray indicate model applications. 


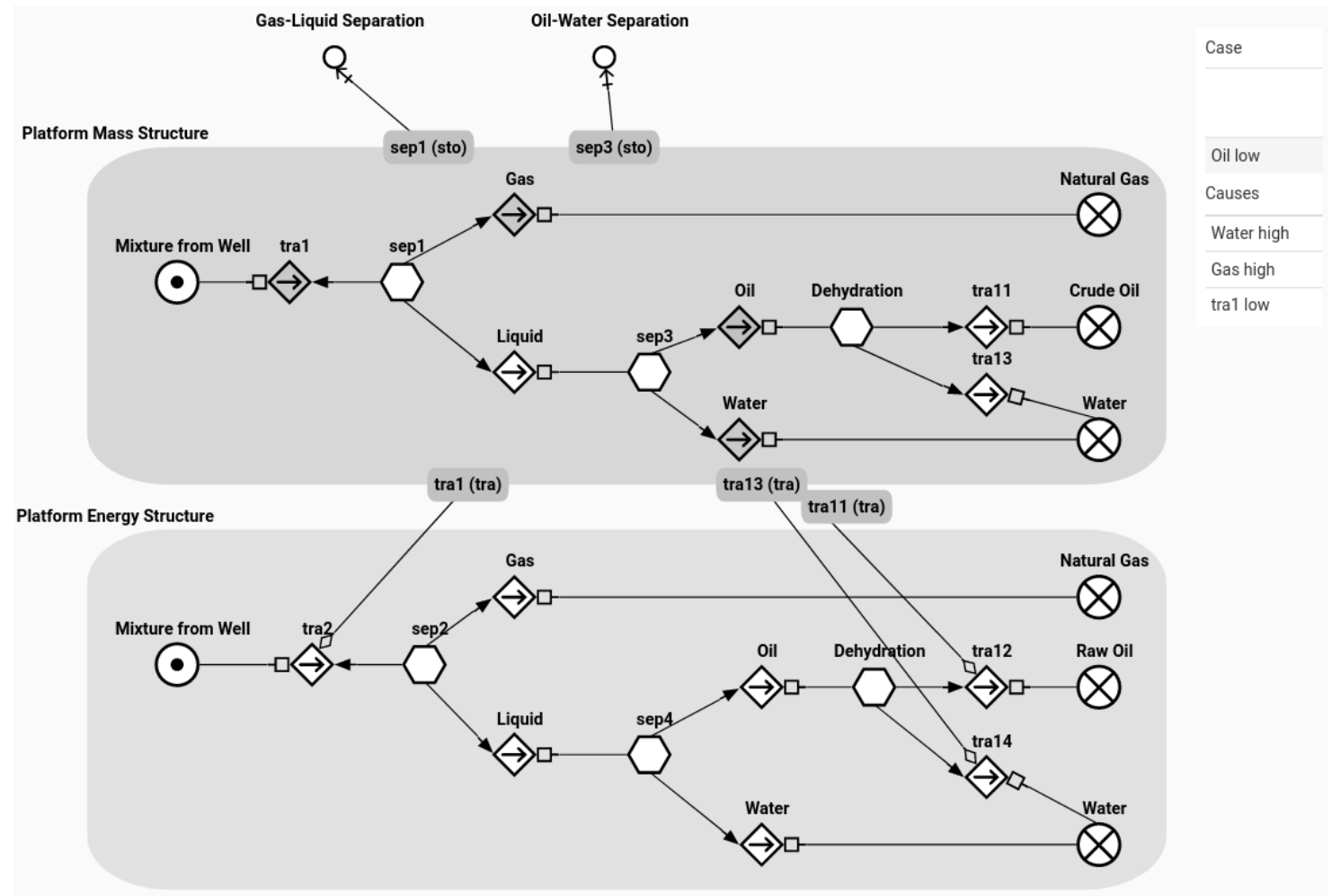

Figure 9: Causal reasoning in the MFM from Figure 7. Potential causes of a low deviation in the oil flow function in the mass flow structure are low flow from the well or high gas flow or high water flow. Darker flow functions indicate potential causes of the deviation. The case and its causes are specified in the text column 


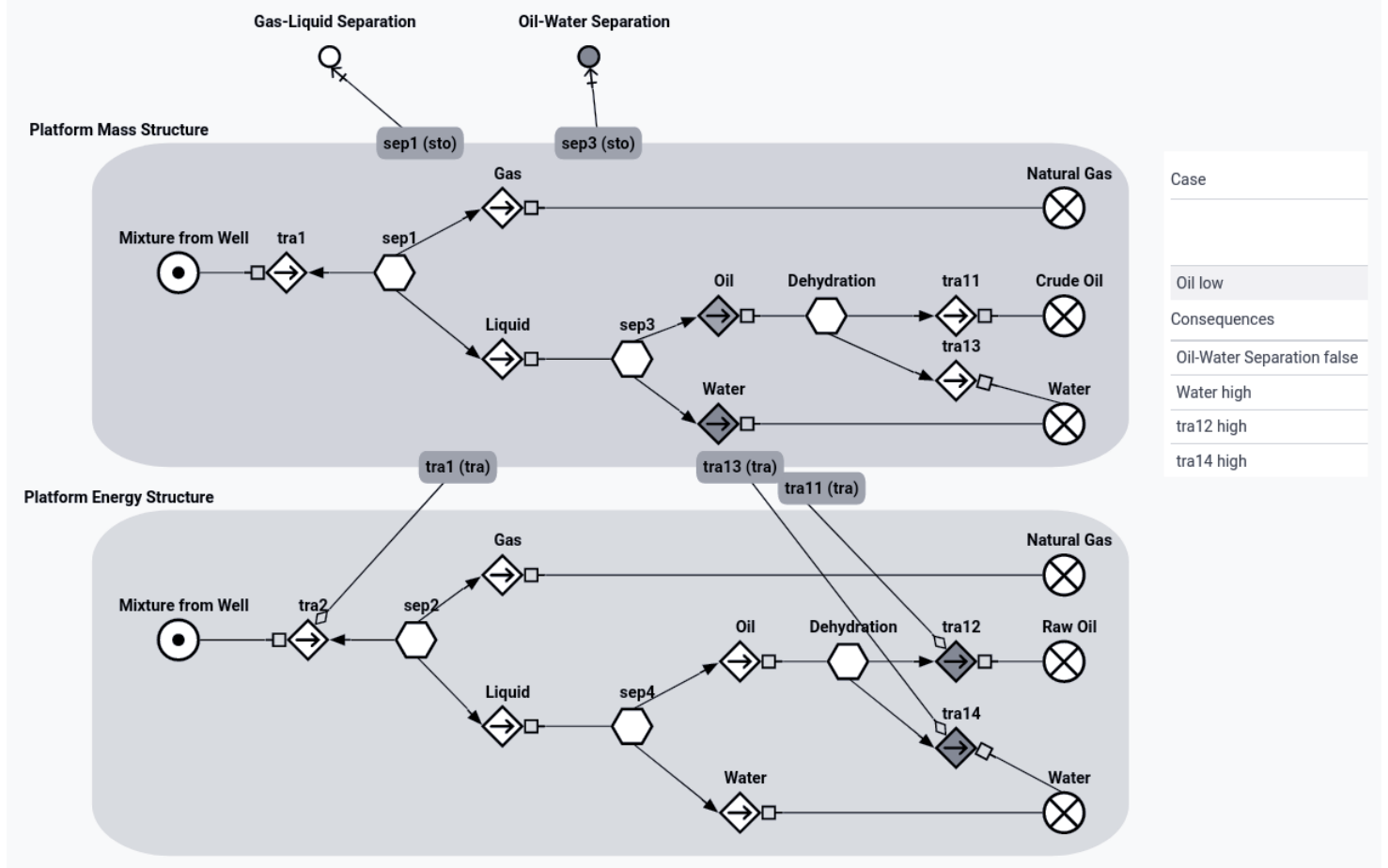

Figure 10: Consequence reasoning in the MFM from Figure 7. Potential consequences of a low deviation in the oil flow function in the mass flow structure are increased water flow and that the oil-water separation objective fails. Darker flow functions indicate potential consequences of the deviation. The case and its consequences are specified in the text column 